\title{
Augmenter of Liver Regeneration Protects against Ethanol-Induced Acute Liver Injury by Promoting Autophagy
}

\author{
Limin Liu, Ping Xie, Wen Li, Yuan Wu, and Wei An
}

From the Department of Cell Biology, Capital Medical University and the Municipal Key Laboratory for Liver Protection and Regulation of Regeneration, Beijing, People's Republic of China

\author{
Accepted for publication \\ November 13, 2018. \\ Address correspondence to \\ Wei An, Ph.D., Department of \\ Cell Biology, Capital Medical \\ University, 10 You An Men \\ Wai Xi Tou Tiao, 100069 \\ Beijing, People's Republic of \\ China. \\ E-mail: anwei@ccmu.edu.cn.
}

\begin{abstract}
Alcoholic liver disease is associated with high morbidity and mortality, and treatment options are limited to date. Augmenter of liver regeneration (ALR) may protect against hepatic injury from chemical poisons, including ethanol. Autophagy appears to positively influence survival in cases of liver dysfunction, although the mechanisms are poorly understood. Herein, we investigated effects of ALR-induced autophagy in vitro and in vivo in an ethanol-induced model of acute liver injury. Decreased serum levels of alanine aminotransferase and aspartate aminotransferase and reduced histologic lesions revealed that mice overexpressing ALR experienced less liver damage than wild-type. ALR-knockdown mice experienced more severe liver damage than wild-type. ALR-transfected HepG2 cells showed increased survival rates, improved maintenance of mitochondrial membrane potential, and increased ATP levels after ethanol treatment. The observed protection was associated with up-regulation of autophagy-markers, including light chain 3II, beclin-1, and autophagy-related gene 5, and down-regulation of p62 by ALR. Autophagy was inhibited in ALR-knockdown mice and HepG2 cells, and autophagy inhibitor bafilomycin A1 attenuated the protective effects of ALR. Results showed phosphorylated mammalian target of rapamycin (mTOR) was down-regulated when ALR was overexpressed and up-regulated when ALR was knocked down. These data show that ALR is protective against ethanol-induced acute liver injury by promoting autophagy, probably via repressing the mTOR pathway. These results have potential implications for the clinical treatment of alcoholic liver disease patients. (Am J Pathol 2019, 189: 552-567; https://doi.org/10.1016/j.ajpath.2018.11.006)
\end{abstract}

Alcoholic liver disease causes approximately two million deaths per year worldwide, and the only treatment option in practical use is corticosteroids. ${ }^{1}$ The spectrum of alcoholmediated liver damage includes steatosis, alcoholic hepatitis, fibrosis, cirrhosis, and hepatocellular carcinoma. ${ }^{2,3}$ Ethanol is converted to acetaldehyde by alcohol dehydrogenase or metabolized by cytochrome P450 2E1 to produce reactive oxygen species (ROS). ROS are toxic because they react with most cellular macromolecules to inactivate intracellular enzymes or denature proteins, cause peroxidization of membrane-bound lipids, destroy membrane integrity, and cause DNA mutations. This reactivity ultimately results in mitochondrial impairment and hepatocyte damage. . $^{4,5}$

Mitochondria are highly susceptible to ethanol damage and may produce large amounts of ROS themselves. They also release proapoptotic proteins that cause hepatocellular death and liver injury. Removal of ethanol-damaged mitochondria plays an important role in the protection of liver cells against alcohol-mediated damage. ${ }^{6}$ Mitophagy, a subtype of autophagy, represents a selective degradation process for dysfunctional or structurally defective mitochondria, ${ }^{7-9}$ which is protective against alcohol-induced liver injury. ${ }^{10-12}$

Augmenter of liver regeneration (ALR; official gene name, GFER) is a liver growth-promoting factor that was initially described in the livers of weanling rats by LaBrecque and Pesch in $1975 .^{13}$ ALR has been shown to protect against acute liver injury caused by carbon tetrachloride, ${ }^{14}$

Supported by National Natural Science Foundation of China grant 31371169.

L.L. and P.X. contributed equally to this work.

Disclosures: None declared. 
D-galactosamine, ${ }^{15}$ hydrogen peroxide, ${ }^{16}$ and cadmium. ${ }^{17}$ ALR has previously been shown to inhibit fibrosis, delaying the onset of thioacetamide-induced cirrhosis in mice. ${ }^{18}$

The native ALR is approximately $23 \mathrm{kDa}$, it resides primarily in the mitochondrial intermembrane space, ${ }^{19}$ and it is important for the survival of hepatocytes because it is associated with the maintenance of mitochondrial membrane stability and mitochondrial permeability transition. ${ }^{20,21}$ Although ALR-mediated protection of mitochondrial morphology and function has been investigated extensively, the effects of ALR on mitophagy are less well understood to date. Our aim was to determine whether ALR-mediated protection against alcoholic liver injury is associated with changes in autophagy, particularly mitophagy. The in vivo and in vitro results revealed that ALR attenuates ethanol-induced hepatotoxicity by activation of autophagy. The protection is probably mediated by inactivation of the Akt/mammalian target of rapamycin (mTOR) pathway, counteracting the inhibition of autophagy caused by ethanol. Our results reveal a novel role of ALR as a mitophagy-promoting factor, which may be useful in the future to treat acute alcoholic liver damage.

\section{Materials and Methods}

\section{Animal Model of Ethanol-Induced Acute Liver Injury}

Male C57BL/6 mice, weighing $18 \mathrm{~g}$ to $22 \mathrm{~g}$, were purchased from the Academy of Military Medical Sciences (Beijing, China) and maintained at constant room temperature $\left(22^{\circ} \mathrm{C}\right.$ to $25^{\circ} \mathrm{C}$ ) on a 12:12-hour light-dark cycle. All animals received humane care following the NIH's Guide for the Care and Use of Laboratory Animals ${ }^{22}$ approved by the Capital Medical University (Beijing, China). To achieve in vivo ALR gene transfection, $1 \times 10^{9}$ plaque-forming units of an ALR plasmid were injected via the tail vein 5 days before ethanol gavage. A pcDNA3.0 vector (Invitrogen, New York, NY) control was injected into the tail vein at the same time. Heterologous ALRknockdown mice (designated as $\mathrm{ALR}^{+/-}$) were generated and maintained in the study center laboratory. The acute ethanol liver injury model was performed, as previously described. ${ }^{11}$ Mice were given a solitary bolus of 55\% ethanol by gastric gavage at a dose of $10 \mathrm{~mL} / \mathrm{kg}$ in the morning. Control animals received the same volume of saline solution. Animals were sacrificed 12 hours after ethanol administration, whole livers were immediately prepared for histologic analysis, some materials were stored at $-80^{\circ} \mathrm{C}$ for RNA preparation, and blood samples were collected for biochemical assays.

\section{Cell Culture, Plasmid Transfection, and Ethanol Treatment}

HepG2 cells, a human hepatoma cell line, were cultured in Dulbecco's modified Eagle's medium with $10 \%$ fetal calf serum in a $37^{\circ} \mathrm{C}$ incubator with $5 \% \mathrm{CO}_{2}$. All culture reagents were purchased from HyClone Laboratories, Inc. (Logan, UT). HepG2 cells were stably transfected with either a pcDNA3.0Fiag-ALR plasmid or a pcDNA3.0 vector as a control and were treated for 12 hours with ethanol at a final concentration of $200 \mathrm{mmol} / \mathrm{L}$ to induce cell injury. Bafilomycin A1 (SigmaAldrich, St. Louis, MO), which inhibits autophagy by inhibiting vacuolar $\mathrm{H}^{+}$-ATPase, was added to the culture medium at a final concentration of $10 \mathrm{nmol} / \mathrm{L} 1$ hour before ethanol administration.

\section{ALR-shRNA}

PLVX-shRNA-ALR-puro was constructed and stably transfected into HepG2 cells to knock down the ALR expression. The shRNA-ALR forward sequence is 5'-GATCCGAAGCGGGACACCAAGTTTAGTTCAAGAGACTAAACTTGGTGTCCCGCTTCTTTTTTCTCGAGG-3'; the shRNAALR reverse sequence is $5^{\prime}$-AATTCCTCGAGAAAAAGAAGCGGGACACCAAGTTTAGTCTCTTGAACTAAACTTGGTGTCCCGCTTCG-3'.

\section{Histologic Evaluation and Biochemical Assays}

Liver specimens were fixed in $10 \%$ neutral-buffered formalin and embedded in paraffin, divided into sections (5 $\mu \mathrm{m}$ thick), and stained with hematoxylin and eosin for histologic examination by light microscopy (DM5000 B; Leica Microsystems, Wetzlar, Germany). Liver function was assayed by the serologic activities of alanine aminotransferase and aspartate aminotransferase by an autoanalyzer (7600-020; Hitachi, Tokyo, Japan) in the Clinical Chemistry Laboratory of Capital Medical University.

\section{Real-Time Quantitative PCR}

Total RNA was isolated and converted to cDNA using the ReverTra Ace (TRT-101; Toyobo, Osaka, Japan). Real-time quantitative PCRs were performed using SYBR Green PCR master mix (QPK201; Toyobo). Primers used in these analyses were as follows: superoxide dismutase (SOD) 2, 5'-GCTCCGGTTTTGGGGTATCTG-3' (forward) and 5'-GCGTTGATGTGAGGTTCCAG-3' (reverse); SOD1, 5'-GGTGGGCCAAAGGATGAAGAG-3' (forward) and 5'GCGTTGATGTGAGGTTCCAG-3' (reverse); and glyceraldehyde-3-phosphate dehydrogenase, 5'-GGGAAGGTGAAGGTCGGAGT-3' (forward) and 5'-TTGAGGTCAATGAAGGGGTCA-3' (reverse).

\section{Luciferase Reporter Assay}

The -52 to -1500 bp sequence in $5^{\prime}$ untranslated region of SOD2 promoter sequence was cloned into the pGL3-Basic vector. Luciferase activity was measured by the Dual Luciferase Assay System (Promega, Madison, WI).

\section{SOD Activity Assay}

Cytosolic and mitochondrial compartments were prepared using a commercial mitochondrial fractionation isolation kit 
(APPLYGEN, Beijing, China). The activity of SOD was determined with the Superoxide Dismutase Activity Assay Kit (Beyotime Biotechnology, Shanghai, China) following the manufacturer's instructions.

\section{Western Blot Assay}

Protein concentrations of cell and tissue lysates were determined with a bicinchoninic acid assay kit (Pierce Biotechnology, Rockford, IL). Aliquots containing $50 \mu \mathrm{g}$ of protein were separated by $8 \%, 12 \%$, or $15 \%$ SDS-PAGE, as needed. Anti-light chain 3 (LC3), anti-p62, anti-autophagy-related gene 5 (Atg5), anti-beclin-1, anti-phosphorylated mTOR (p-mTOR), anti-total-mTOR, anti-phosphorylated Akt, anti-Akt, anti-ALR primary antibodies (all at 1:1000), and anti- $\beta$-actin (at 1:5000), all from Cell Signaling Technology (Danvers, MA), were used. The relative density of protein bands was quantitated with ImageJ version 1.5s (http://imagej. nih.gov/ij, last accessed June 28, 2018) provided in the public domain by the NIH (Bethesda, MD).

\section{Confocal Microscopy}

Cells were seeded in culture dishes specialized for confocal microscopy and stained with Hoechst 33432 to label the nuclei after transfection with adenovirus-red fluorescent proteingreen fluorescent protein-light chain 3 . Frozen sections were blocked with $10 \%$ bovine serum albumin at room temperature for 1 hour and incubated with primary antibodies (anti-LC3II antibody at 1:200; Proteintech, Chicago, IL) at $4{ }^{\circ} \mathrm{C}$ overnight. Secondary antibody staining was 1 hour at room temperature in an Alexa Fluor 488-conjugated goat anti-rabbit IgG antibody solution (Invitrogen). After nuclear staining with DAPI at $37^{\circ} \mathrm{C}$ for 5 minutes, staining was analyzed and documented using a

\section{A}

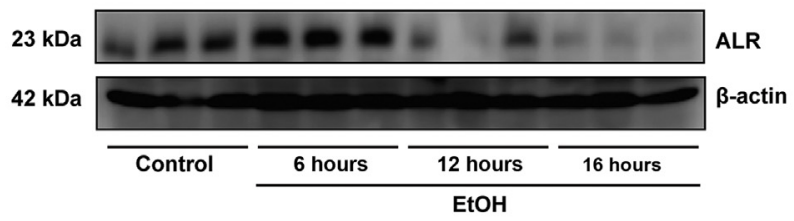

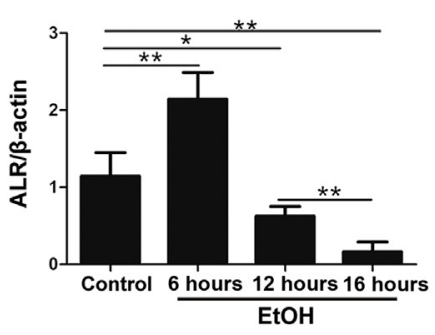

B

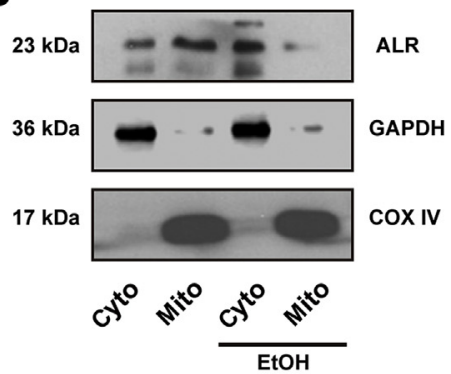

C
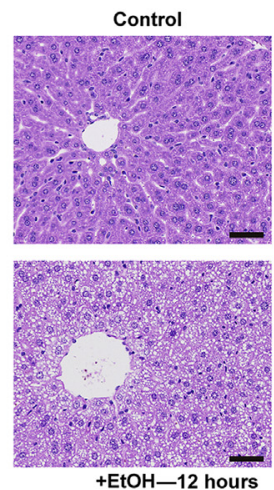

$\mathbf{E}$
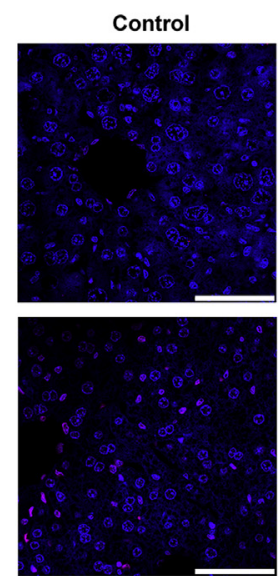

+ EtOH-12 hours
+EtOH-6h

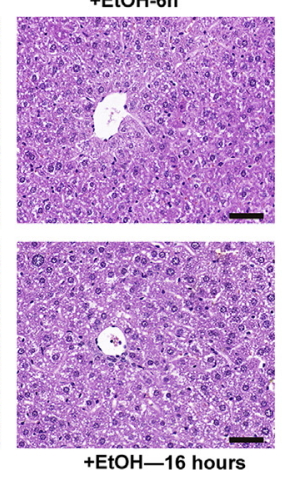

+ EtOH -6 hours
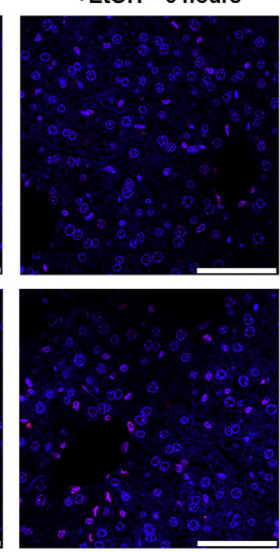

+EtOH-16 hours
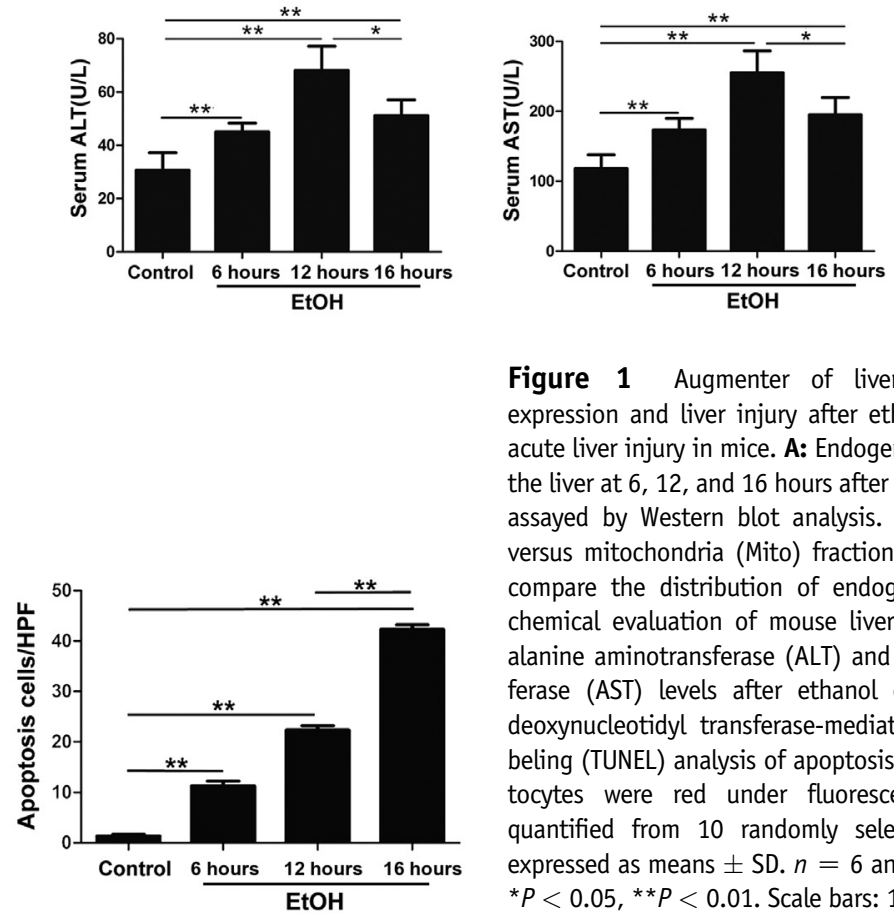

Figure 1 Augmenter of liver regeneration (ALR) expression and liver injury after ethanol $(\mathrm{EtOH})$-induced acute liver injury in mice. A: Endogenous ALR expression in the liver at 6,12 , and 16 hours after ethanol administration assayed by Western blot analysis. B: Cytoplasmic (Cyto) versus mitochondria (Mito) fractionation was analyzed to compare the distribution of endogenous ALR. C: Histochemical evaluation of mouse liver specimens. D: Serum alanine aminotransferase (ALT) and aspartate aminotransferase (AST) levels after ethanol exposure. E: Terminal deoxynucleotidyl transferase-mediated dUTP nick-end labeling (TUNEL) analysis of apoptosis. TUNEL-positive hepatocytes were red under fluorescence microscopy and quantified from 10 randomly selected fields. Data are expressed as means \pm SD. $n=6$ animals $(\mathbf{A}, \mathbf{C}, \mathbf{D}$, and $\mathbf{E})$. ${ }^{*} P<0.05,{ }^{*} P<0.01$. Scale bars: $100 \mu \mathrm{m}(\mathbf{C}) ; 75 \mu \mathrm{m}(\mathbf{E})$. COX, cytochrome c oxidase; GAPDH, glyceraldehyde-3phosphate dehydrogenase; HPF, high-power field. 
A

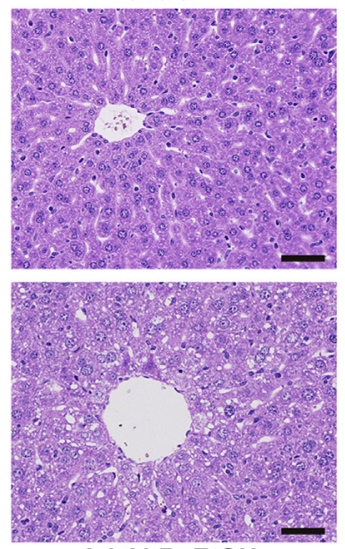

Ad-ALR+EtOH

C

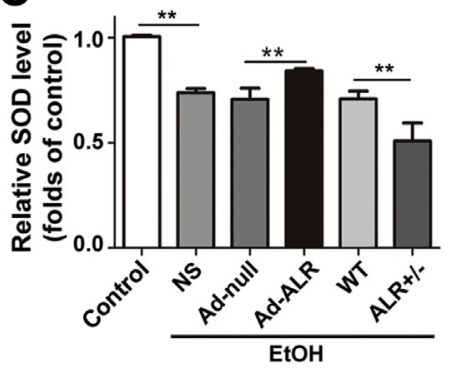

E

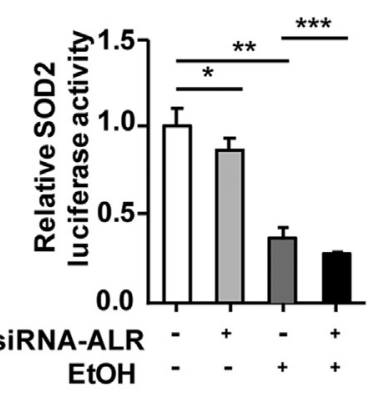

NS+EtOH
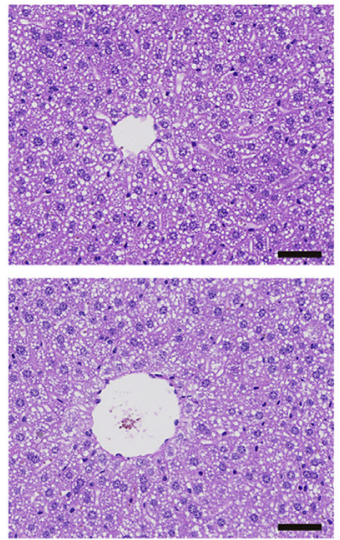

WT+EtOH
Ad-null+EtOH
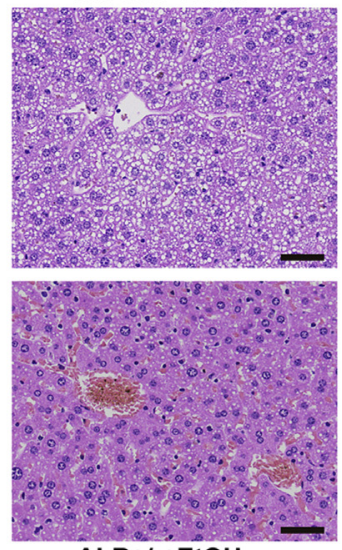

ALR+/-+EtOH
B
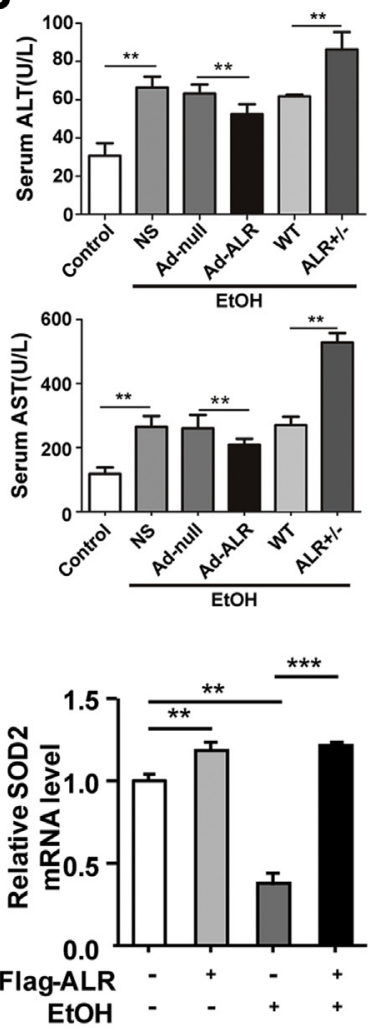

$\mathbf{F}$
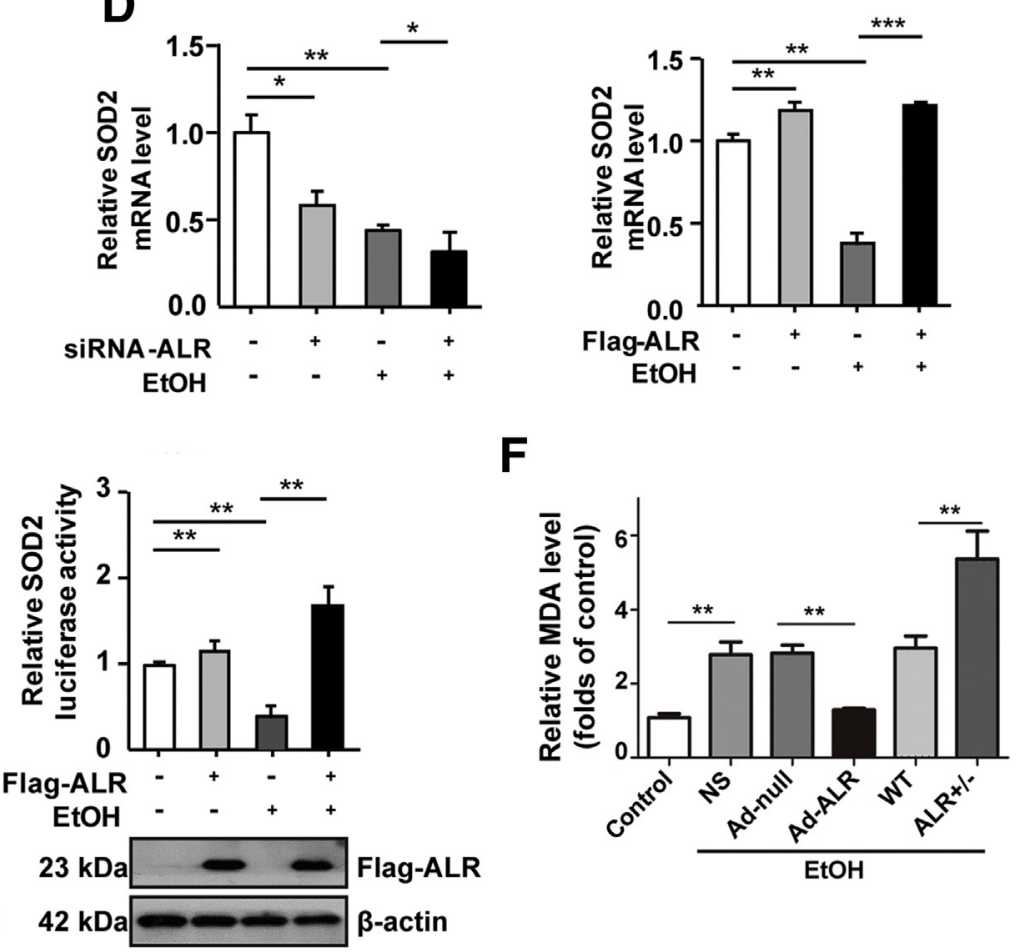

G

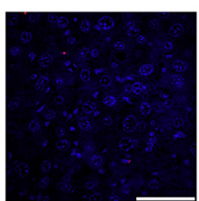

Control

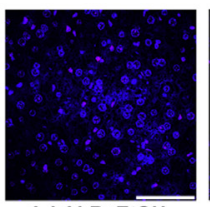

Ad-ALR+EtOH

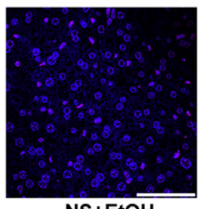

NS+EtOH

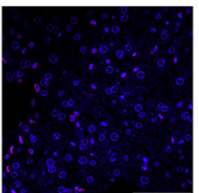

WT+EtOH

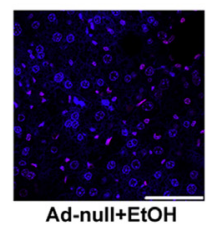

Ad-null+EtoH

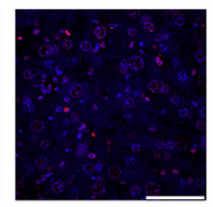

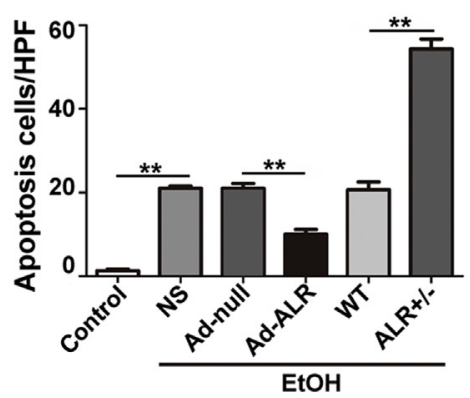

Figure 2 Protective effects of augmenter of liver regeneration (ALR) expression against ethanol (EtOH)-induced acute liver injury in mice. Tissue and serum were obtained from mice sacrificed at 12 hours after ethanol administration. A: Liver tissues stained with hematoxylin and eosin. B: Serum alanine aminotransferase (ALT) and aspartate aminotransferase (AST). C: Hepatic superoxide dismutase (SOD) levels. D: Real-time quantitative PCR analysis of the mRNA level of SOD2 (mitochondrial isoform). E: Promoter activity assay for the SOD2 gene. F: Hepatic malondialdehyde (MDA) levels. G: Terminal deoxynucleotidyl transferase-mediated dUTP nick-end labeling quantification of apoptosis. Data are expressed as means \pm SD. ${ }^{*} P<0.05$, ${ }^{* *} P<0.01$, and $* * * P<0.001$. Scale bars: $100 \mu \mathrm{m}$ (A); $75 \mu \mathrm{m}$ (D). Ad, adenovirus; Flag, FLAG-tag; HPF, high-power field; NS, normal saline; WT, wild type. 
A

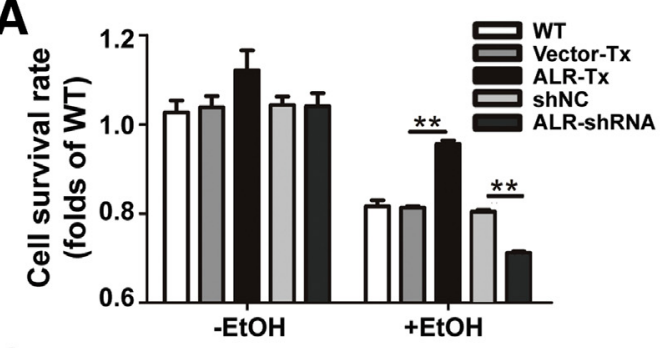

C

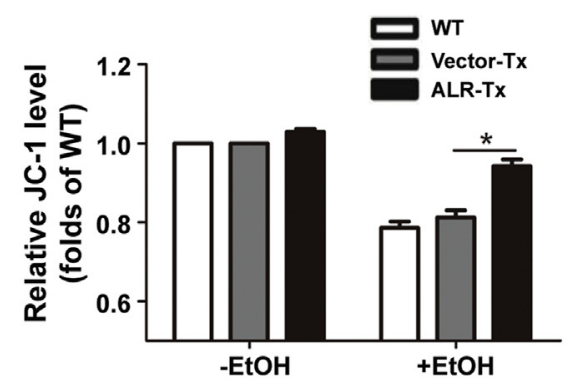

E
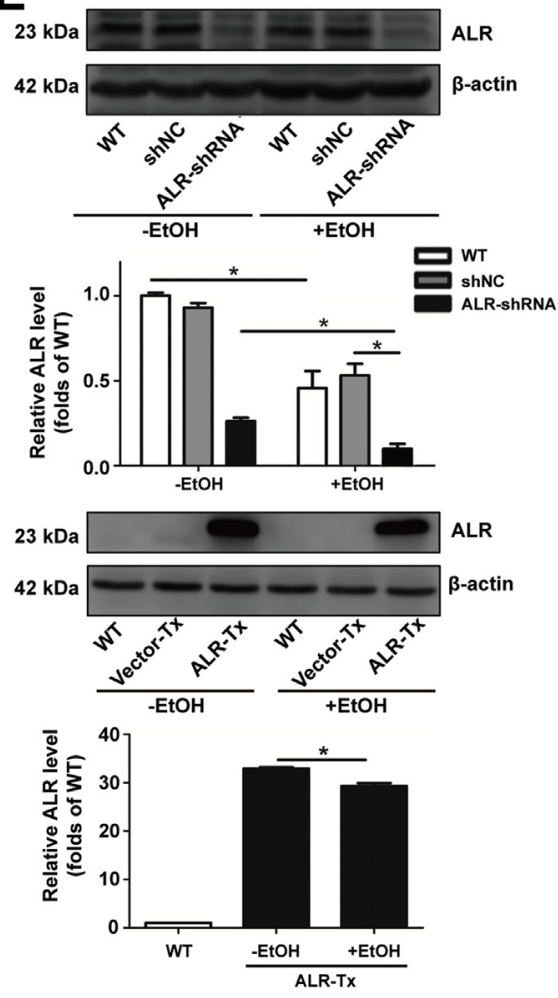

B

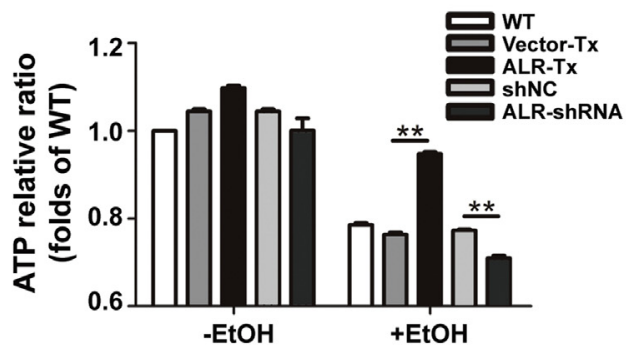

D
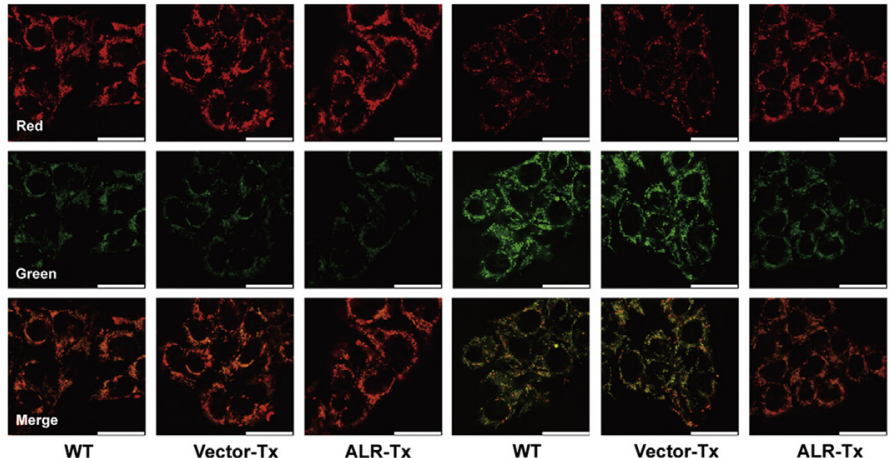

Vector-Tx

ALR-Tx

$\mathbf{F}$
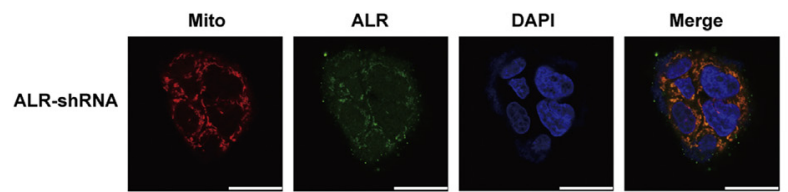

WT
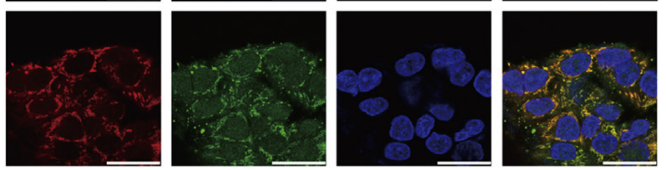

ALR-Tx
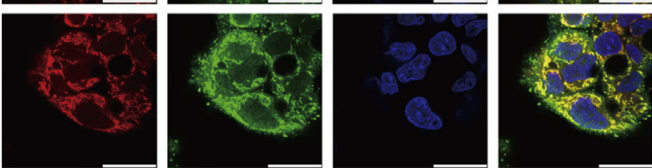

Figure 3 Protective effects of augmenter of liver regeneration (ALR) expression against ethanol (EtOH)-induced liver injury in HepG2cells. A: Cell viability. B: Cellular ATP content. C: Change in the mitochondrial transmembrane potential (MTP) analyzed by high-content screening. D: Change in MTP analyzed by confocal microscopy. E: ALR in ALR-shRNA and ALR-transfected versus control cells before and after alcohol. F: Colocalization of ALR and MitoTracker (Invitrogen) by confocal microscopy. All data were repeated at least three times. Data are expressed as means \pm SD. ${ }^{\star} P<0.05$, ${ }^{* \star} P<0.01$. Scale bar $=25 \mu \mathrm{m}(\mathbf{D}$ and $\mathbf{F})$. shNC, negative control; Tx, treatment; WT, wild type.
Leica NT fluorescent confocal microscope (Leica Microsystems).

\section{Assay of Intracellular ATP}

Intracellular ATP was assayed with a CellTiter-Glo luminescent cell viability assay kit (Promega), following the manufacturer's instructions. After vortex mixing and centrifugation, the ATP contents were immediately measured with a Glomax 96 Microplate Luminometer (Promega).

\section{Measurement of Mitochondrial Transmembrane Potential}

The mitochondrial transmembrane potential (MTP) was assayed using a JC-1 assay (Sigma-Aldrich). JC-1 is a 
molecular probe that forms $\mathrm{J}$-aggregates in the polarized mitochondrial matrix, which emit red fluorescence at $595 \mathrm{~nm}$ when excited at $525 \mathrm{~nm}$. In the depolarized mitochondrial matrix, JC-1 exists as monomers and emits green fluorescence at $525 \mathrm{~nm}$ when excited at $485 \mathrm{~nm}$. Mitochondrial depolarization is visualized by a decrease in the red/green fluorescence intensity ratio. After ethanol treatment, cells were quantitatively analyzed by High Content Screening (ArrayScan XTI; Thermo Fisher Scientific, Waltham, MA) and JC-1 assay kit, following the manufacturer's instructions.

\section{RNA-Seq and Data Analysis}

Total RNA was extracted from samples with TRIzol (15596; Invitrogen), according to the manufacturer's instructions. cDNA libraries were prepared as previously described. ${ }^{23}$ The library products were sequenced on an IlluminaHiSeq 2000 (Illumina, San Diego, CA). Results from reads that could be uniquely mapped to a gene were used to calculate the expression level. Cufflinks ${ }^{24}$ version 2.1.1 was used to obtain gene-based fragments per kilobase of exon per million fragments mapped values for all samples, which corrects for transcript length and the total number of mapped reads from the library to compensate for different read depths for different samples. FASTQC was used to check the quality of reads of all samples (http://www.bioinformatics.babraham. ac.uk/projects/fastqc, last accessed July 16, 2018).

Raw data were filtered to remove reads containing adapter and poly-N and low-quality reads $(\mathrm{Q}<20)$ generating clean data. TopHat2 version 2.0.0 25 was used to map RNA deep sequencing (RNA-Seq) reads to the human reference transcriptome and genome. TopHat2 initially attempts to map reads to the reference transcriptome and subsequently attempts to map unmapped reads to the human genome reference. The expression of each gene was normalized by the reads per kilobase per million mapped reads among different samples. The differences in gene expression were judged using the threshold value of $[\log 2$ (fold change) $] \geq 1$ with a false discovery rate $\leq 0.001$. For enrichment analysis of differentially expressed genes, gene ontology enrichment analysis and Kyoto Encyclopedia of Genes and Genomes (https://www.kegg.jp/kegg/pathway.html, last accessed July 16 , 2018) pathway analysis of differentially expressed genes were performed using the DAVID functional annotation tool ${ }^{26,27}$ (http://david.abcc.ncifcrf.gov, last accessed July 10, 2018). The corrected $P \leq 0.05$ was considered significantly enriched by differentially expressed genes. For RNA sequencing, the transcriptome data sets are available at the National Center for Biotechnology Information Sequence Read Archive (https://www.ncbi.nlm. nih.gov/sra; accession number SRP153001).

\section{Statistical Analysis}

Results were reported as the average of at least three biological replications and expressed as means $\pm \mathrm{SD}$. The significance of between-group differences was tested by $t$-test and two-way analysis of variance. $P<0.05$ denotes a statistically significant difference.

\section{Results}

\section{ALR Involvement in Ethanol-Induced Acute Liver Injury in Mice}

Involvement of ALR in ethanol-induced liver injury was measured by analysis of changes in ALR expression during ethanol administration. Western blot analysis results indicate that endogenous ALR protein expression was significantly down-regulated at 12 and 16 hours of ethanol exposure (Figure 1A). Furthermore, mitochondria isolation assay was performed to detect the subcellular compartmentalization of ALR under ethanol treatment. ALR in mitochondria obviously decreased with the treatment of ethanol, but ALR in cytoplasm was unaffected (Figure 1B). Cell swelling was apparent at both 12 and 16 hours (Figure 1C). Serum alanine aminotransferase and aspartate aminotransferase activities were also increased, with peak levels at 12 hours (Figure 1D). Numbers of apoptotic cells were increased after ethanol injury (Figure 1E). These results indicate that ALR is affected by ethanol-induced acute liver injury.

\section{ALR Transfection Protects Mice from Ethanol-Induced Acute Liver Injury}

To determine whether ALR expression is protective against ethanol-induced liver injury, mice were transfected with ALR-containing viral plasmids by tail vein injection. Exposure to ethanol induced significant hepatic damage compared with the control group (Figure 2A). The hepatic lesions of ALR-transfected mice were markedly attenuated, with decreased cell ballooning, cell degeneration, and cytoplasmic vacuolation. However, the hepatic lesions were worse in $\mathrm{ALR}^{+/-}$mice. Serum alanine aminotransferase and aspartate aminotransferase levels were significantly decreased in ALR-transfected mice and significantly elevated in $\mathrm{ALR}^{+/-}$mice (Figure 2B). SOD, which functions to remove ROS, was maintained in the livers of ALRtransfected mice exposed to ethanol, compared with that in $\mathrm{ALR}^{+/-}$mice (Figure 2C). To determine whether the decrease of SOD occurs at the transcriptional step, real-time quantitative PCR was performed to detect the transcriptional activity of SOD2 (mitochondrial isoform). The treatment of ethanol clearly decreases the mRNA level of SOD2, whereas the ALR-siRNA exacerbates the reduction in SOD2. On the contrary, overexpression of ALR prevents mitochondrial SOD from further decline on ethanol injury (Figure 2D). To determine whether ALR regulates the activity of SOD2 promoter, a luciferase reporter assay is used. A segment containing the -52 to $-1500 \mathrm{bp}$ of $S O D 2$ gene promoter sequences is constructed into pGL3 basic vector. 
A

B

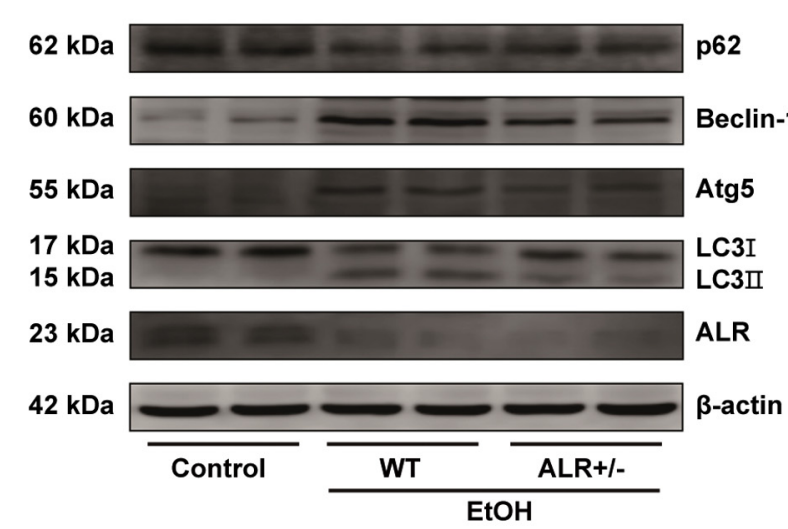

C

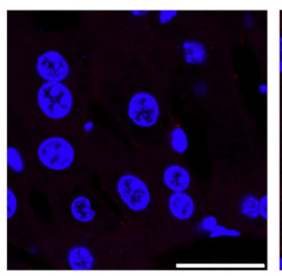

Control

D

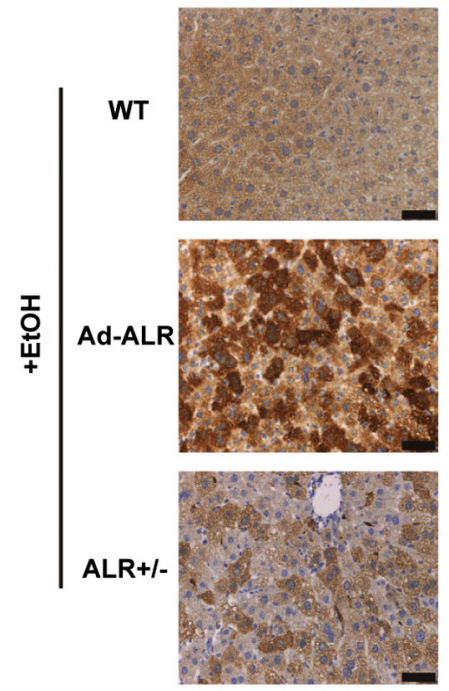

ALR

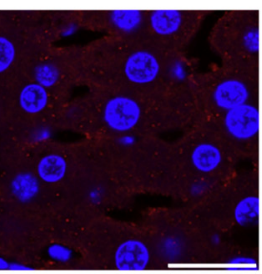

NS+EtOH

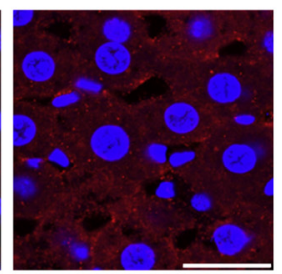

Ad-null+EtOH
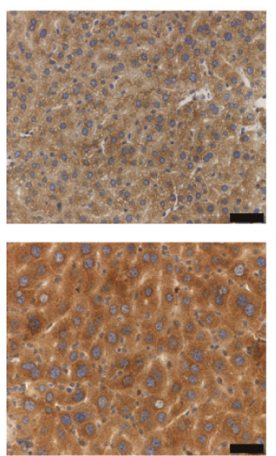

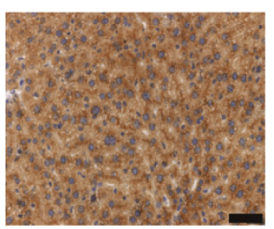

mTOR
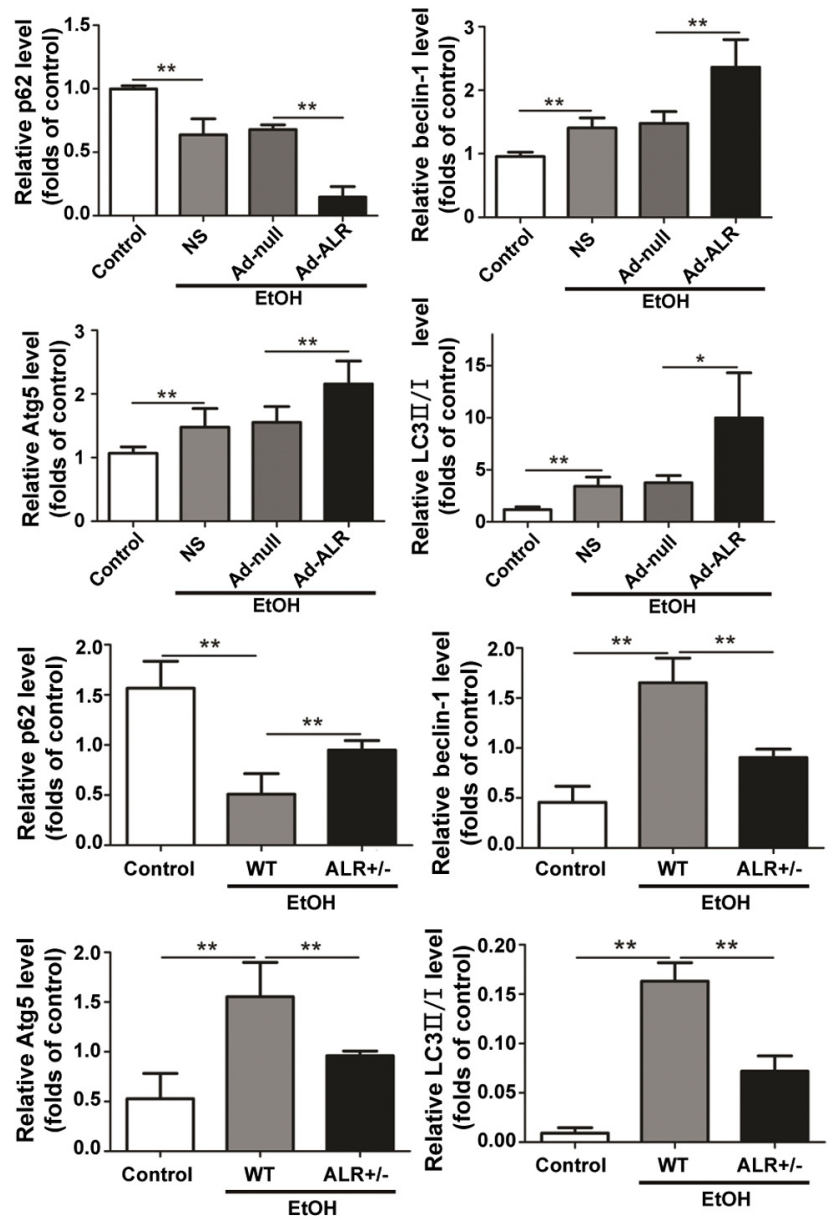

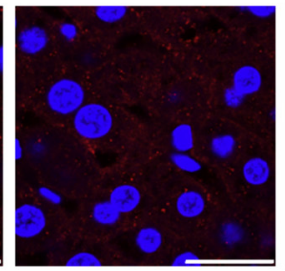

WT+EtOH

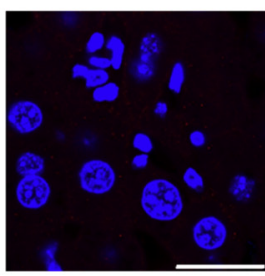

ALR+/-+EtOH

Ad-ALR+EtOH
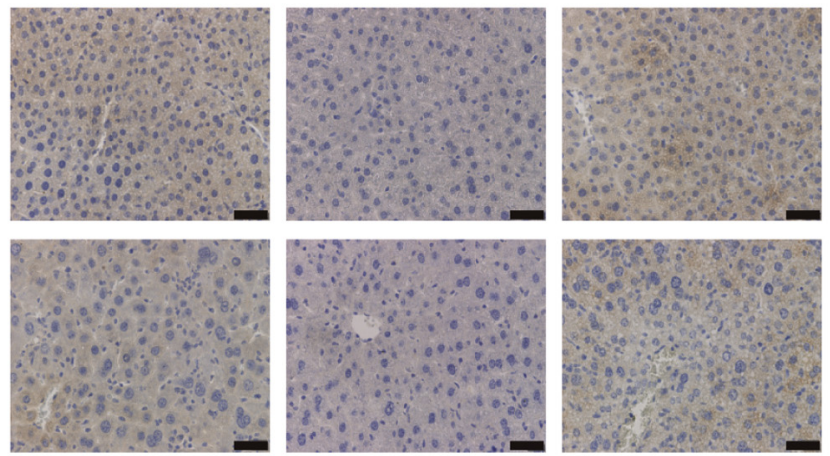

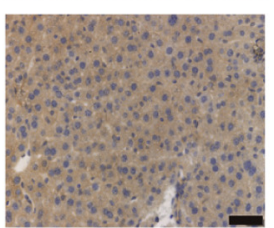

p-mTOR

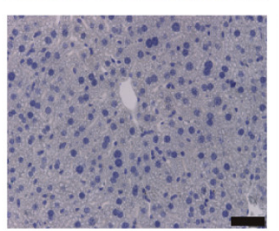

LC3-b
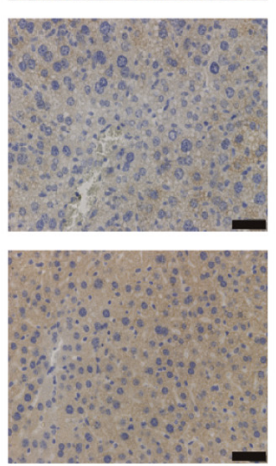

p62 
The result of reporter assay indicated that there is distinct down-regulation of SOD2 transcription on the treatment of ethanol, the ALR-siRNA deteriorates the down-regulation of SOD2 promoter, and overexpression of ALR abolishes this inhibition (Figure 2E). Real-time quantitative RT-PCR was performed to investigate the effect of ALR on transcriptional activity of SODI (cytosolic SOD), and it was found that ALR did not affect the mRNA level of SOD1. Collectively, these results strongly suggest ALR promotes the mitochondrial SOD at transcriptional level to combat ethanol-induced hepatocyte injury. Abundance of malondialdehyde, a natural biproduct of lipid peroxidation used as a marker for lipid peroxidation, increased in vectortransfected mice after ethanol treatment, but decreased in ALR-transfected mice. ALR deletion resulted in a sharp increase of malondialdehyde (Figure 2F). Cells were labeled with terminal deoxynucleotidyl transferasemediated dUTP nick-end labeling as to quantify apoptosis. After ethanol injury, considerable numbers of terminal deoxynucleotidyl transferase-mediated dUTP nick-end labeling-positive cells were identified in livers of $A L R^{+/-}$mice (Figure 2G). These results indicate that ALR expression protects the livers of mice against ethanolinduced injury.

\section{ALR Protects HepG2 Cells against Ethanol-Induced Acute Injury}

To determine whether ALR protected hepatocytes from ethanol-induced acute liver injury in vitro, cell viability was evaluated using a Cell Counting Kit (CCK-8; Dojindo Laboratories, Kumamoto, Japan). The exposure of cells to 200 $\mathrm{mmol} / \mathrm{L}$ ethanol for 12 hours caused detectable cell death (Figure 3A). The survival of ALR-transfected cells was significantly higher than vector-transfected cells, whereas cell survival declined when ALR expression was knocked down by shRNA. These results show that ALR expression increases the hepatocytes' resistance to ethanol injury.

Mitochondria are susceptible to ethanol insult, resulting in severe morphologic and functional impairment. The MTP was used as an indicator of mitochondrial membrane integrity to investigate ethanol-induced mitochondrial dysfunction. The MTP assay measured the ratio of red/green JC-1 fluorescence intensity. Ethanol treatment led to an increase in green-stained mitochondria (ie, depolarization and loss of MTP). Loss of MTP after ethanol treatment was significantly reduced in ALR-transfected cells (Figure 3, C and D). Meanwhile, the ATP content significantly decreased after ethanol treatment, and the decrease in ATP content was significantly lower in
ALR-transfected cells (Figure 3B). Meanwhile, ALR in ALRshRNA cells was much lower than in wild-type (WT) cells after alcohol treatment. At the same time, ALR in ALRtransfected cells was also decreased after alcohol treatment, and obviously higher than control cells (Figure 3E). In light of ALR loss, a decrease in ALR was specifically seen in mitochondria in ALR-shRNA; on the contrary, there was an increase in ALR-transfected cells (Figure 3F). These results indicate that ALR expression attenuates the loss of mitochondrial function and morphology after ethanol insult.

\section{ALR Promotes Autophagy in Ethanol-Induced Acute Liver Injury in Vivo}

Microtubule-associated protein LC3 and p62 were used as markers of autophagy, in which the conversion of LC3I to LC3II indicates the closure of autophagic vacuoles and p62 degradation indicates the fusion of autophagosomes and lysosomes. ${ }^{28}$ Beclin-1 and Atg5 participate in the initiation and extension of autophagic vesicles during autophagosome. ${ }^{29}$ LC3I conversion to LC3II was increased in ALR-transfected mice. Similarly, Atg5 and beclin-1 were elevated after ethanol, but p62 decreased (Figure 4A). In $\mathrm{ALR}^{+/-}$mice, the ratios between LC3II and LC3I, Atg5, and beclin-1 expression were reduced, but p62 expression was enhanced compared with WT mice (Figure 4B). In addition, LC3II red fluorescence spots were increased in the livers of ALR-transfected mice (Figure 4C). Moreover, after the treatment of ethanol, ALR knockdown mice enhanced the phosphorylation of mTOR and the level of p62, but attenuated the LC3II expression, and the reverse effect was found in ALR-transfected mice (Figure 4D). These results indicate that ALR participates in the activation of autophagy in mice with ethanol-induced liver injury.

\section{ALR Augments Both Autophagy and Mitophagy in Ethanol-Injured HepG2 Cells}

Ethanol-induced hepatic autophagy at the cellular level was assessed in ALR-transfected or ALR-shRNA HepG2 cells. ALR transfection promoted LC3II conversion and p62 degradation, and the expression of Atg5 and beclin-1 in ALR-transfected cells was up-regulated after ethanol treatment (Figure 5A). The expression profiles of these autophagy-related proteins in ALR-shRNA cells were similar to those found in the liver of ALR-knockdown mice (Figure 5B). The fluorescent puncta observed in ALRtransfected cells indicated that autophagy was enhanced after ethanol treatment (Figure 5C). These in vitro results confirm that recombinant ALR expression enhances autophagy in hepatocytes after ethanol insult.

Figure 4 Augmenter of liver regeneration (ALR) transgene expression-mediated promotion of autophagy in mice with ethanol (EtOH)-induced liver injury. A: Western blot analyses and density ratios of LC3II/I, p62, autophagy-related gene 5 (Atg5), and beclin-1 in mice overexpressing ALR and control animals. B: Western blot analyses and density ratios of LC3II/I, p62, Atg5, and beclin-1 in ALR ${ }^{+/-}$mice and wild-type controls. C: Histochemical immunofluorescence staining of LC3II in liver tissue from the study groups. D: Immunohistochemical staining to detect the level of mammalian target of rapamycin (mTOR), phosphorylated mTOR (p-mTOR), p62, LC3II, and ALR. Data are expressed as means \pm SD. $n=6$ animals. ${ }^{*} P<0.05,{ }^{* *} P<0.01$. Scale bars: $25 \mu \mathrm{m}$ (C); $40 \mu \mathrm{m}$ (D). Ad, adenovirus; NS, normal saline. 

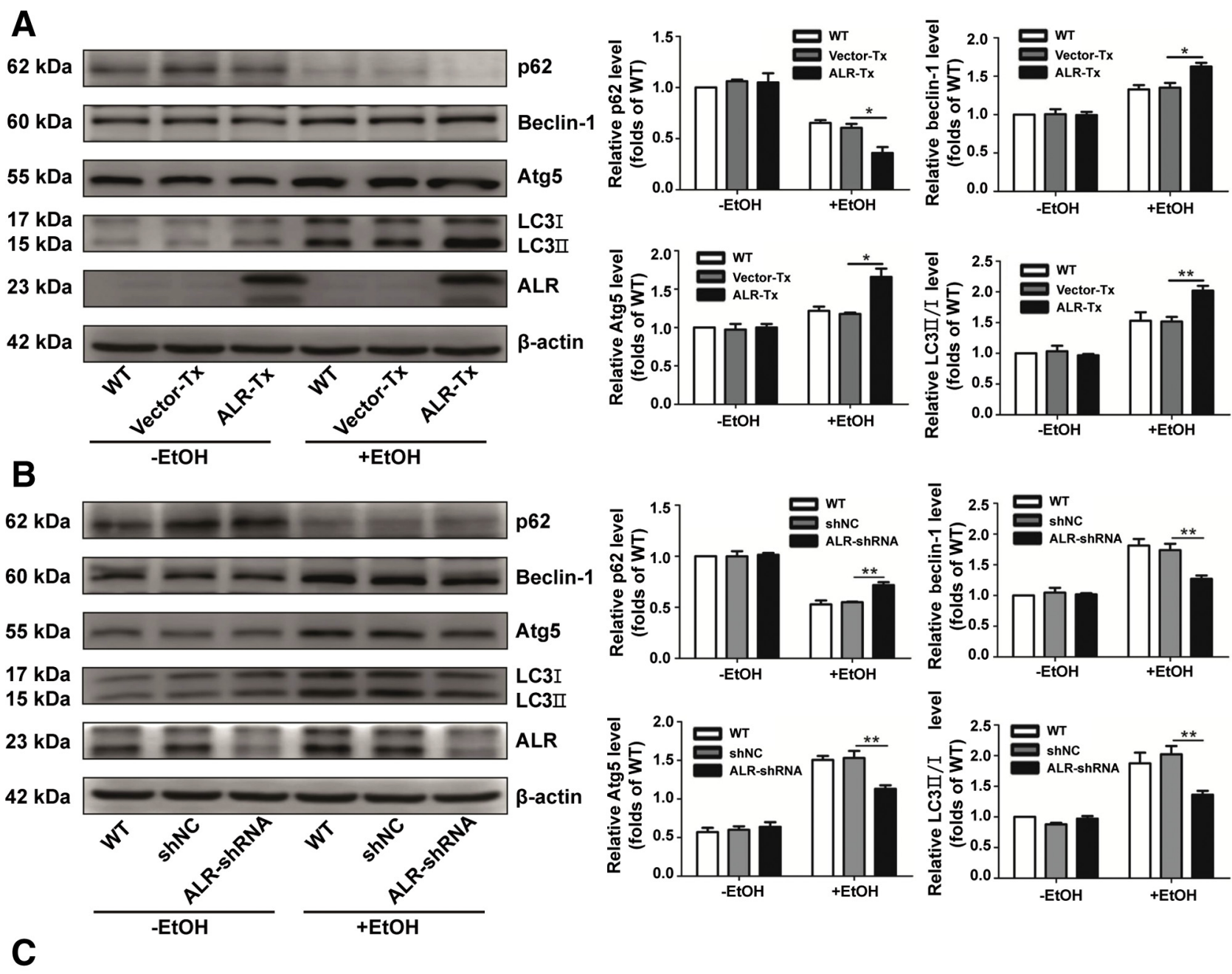

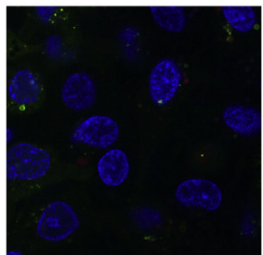

WT

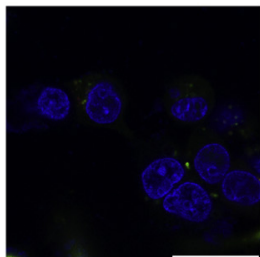

Vector-Tx

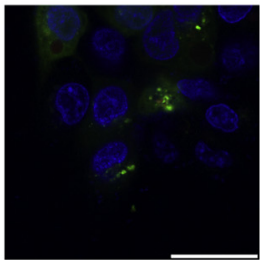

ALR-Tx

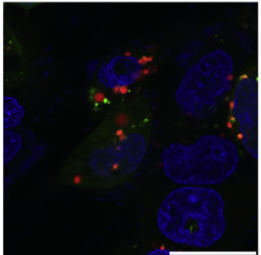

WT

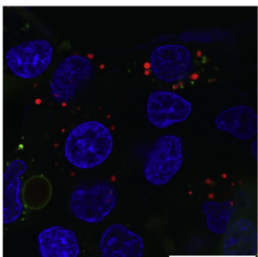

Vector-Tx

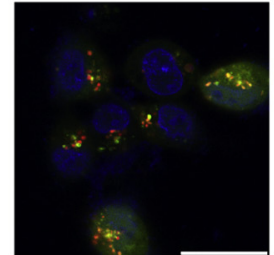

ALR-Tx

Figure 5 Augmenter of liver regeneration (ALR) expression mediates promotion of autophagy in HepG2 cells treated with ethanol. A: Western blot analyses and density ratios of LC3II/I, p62, autophagy-related gene 5 (Atg5), and beclin-1 in the ALR-transgene and control cells. B: Western blot analyses and density ratios of LC3II/I, p62, Atg5, and beclin-1 in the ALR-shRNA and control cells. C: LC3 intracellular fluorescence puncta after ethanol (EtOH) administration. Data are expressed as means \pm SD. ${ }^{*} P<0.05,{ }^{*} P<0.01$. Scale bar $=25 \mu \mathrm{m}(\mathbf{C})$. shNC, negative control; Tx, treatment; WT, wild type.

Mitophagy selectively removes dysfunctional or damaged mitochondria to maintain a healthy mitochondria population. ${ }^{8,9}$ The translocase of outer mitochondrial membrane complex facilitates protein transport through the outer mitochondrial membrane. Translocase of outer mitochondrial membrane 20, a translocase of outer mitochondrial membrane complex receptor, decreases when mitophagy occurs because it is degraded. ${ }^{30}$ Translocase of outer mitochondrial membrane 20 expression in ALR-transfected cells significantly decreased after ethanol treatment, but was significantly increased in ALR-shRNA cells compared with control cells (Figure 6, A and B).

Parkin is an evolutionarily conserved E3 ubiquitin ligase, which is believed to be required for initiation of mitophagy in an in vitro model. ${ }^{31}$ During mitochondrial depolarization, phosphatase and tensin homolog-induced putative kinase 1 is stabilized in the outer mitochondrial membrane, where it phosphorylates and recruits Parkin in damaged mitochondria. Ethanol treatment enhanced the translocation of Parkin to mitochondria, and the translocation was prominent in 
A
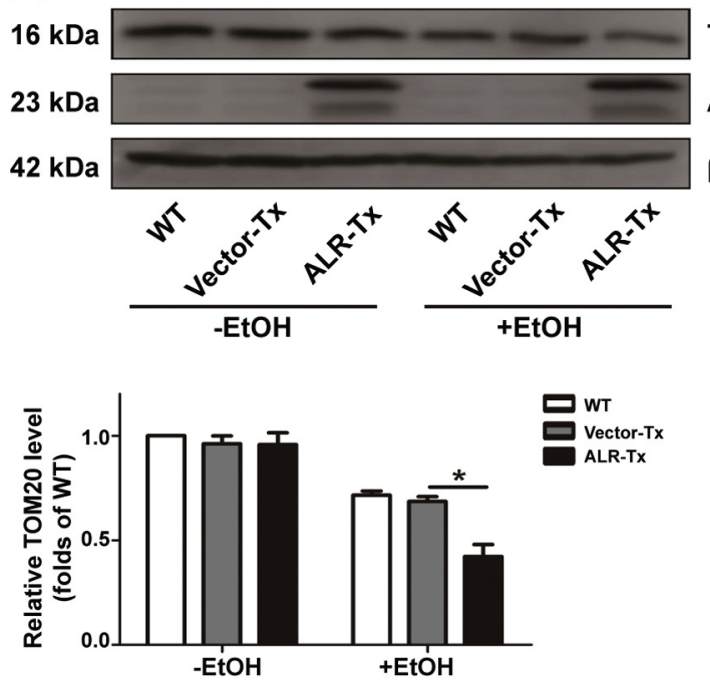

C
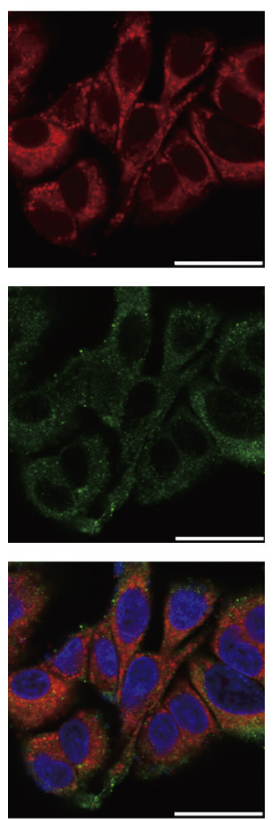

WT
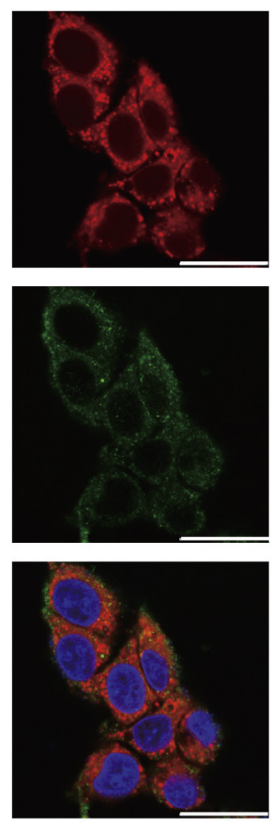

Vector-Tx
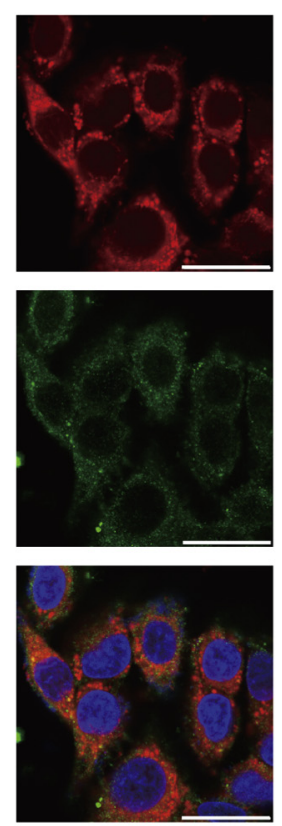

ALR-TX
TOM20

ALR

$\beta$-actin

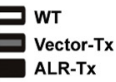

B
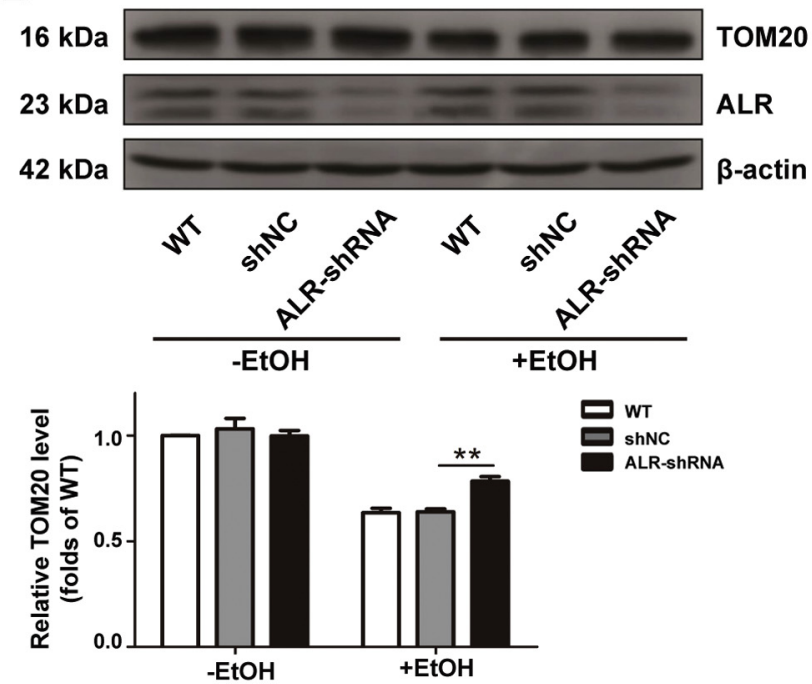

wT

shNC

ALR-shRNA
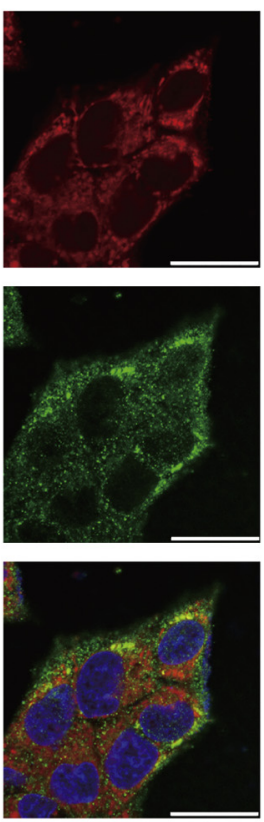

WT
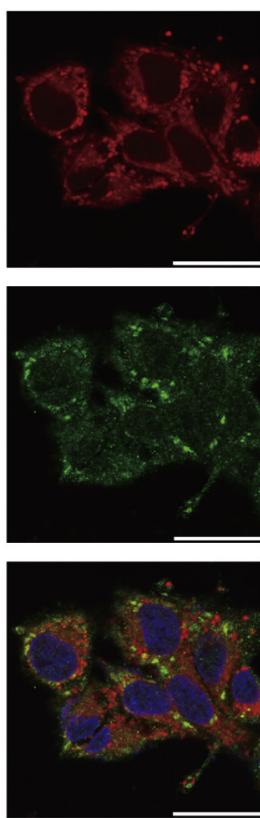

Vector-Tx
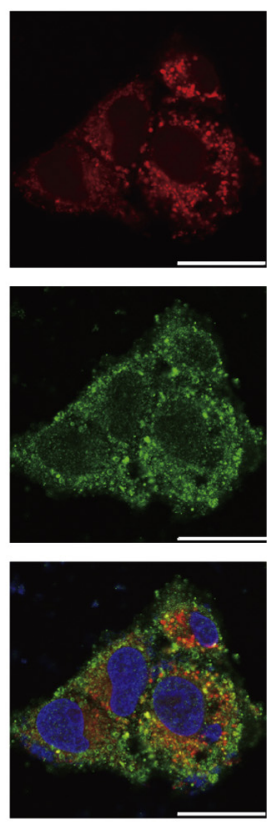

ALR-Tx

Figure 6 Augmenter of liver regeneration (ALR) expression promotes mitophagy. A: Western blot analyses and density ratios of translocase of outer mitochondrial membrane (TOM) 20 in the ALR-transgene and control cells. B: Western blot analyses and density ratios of TOM20 in the ALR-shRNA knockdown and control cells. C: Ethanol (EtOH)-induced Parkin translocation to the mitochondria in HepG2 cells. Parkin (green) and MitoTracker (red; Invitrogen) were immunolabeled, and nuclei were stained with Hoechst 33242. Yellow shows colocalization of Parkin and MitoTracker, indicating Parkin translocation to the mitochondria. The assays were repeated at least three times. Data are expressed as means \pm SD. ${ }^{*} P<0.05,{ }^{* *} P<0.01$. Scale bar $=25 \mu \mathrm{m}(\mathrm{C})$. shNC, negative control; Tx, treatment; WT, wild type.

ALR-transfected cells compared with vector-transfected controls (Figure 6C).

\section{ALR Hepatic Protection Is Partially Related to} Autophagy in HepG2 Cells

BafilomycinA1 (BafA1) is a macrolide antibiotic that reversibly inhibits late-phase autophagy through inactivation of $\mathrm{H}^{+}$-ATPase and thereby blocks fusion of autophagosomes and lysosomes. ${ }^{32-34}$ Western blot assays, which were used to test if inhibition of autophagy by BafA1 could be aggravated in ALR-transfected cells, revealed that LC3II and p62 expression increased in a dose-dependent manner (Figure 7A); $10 \mathrm{nmol} / \mathrm{L}$ BafA1 inhibited autophagy. Levels of LC3II and p62 were significantly increased by BafA1 treatment, suggesting 

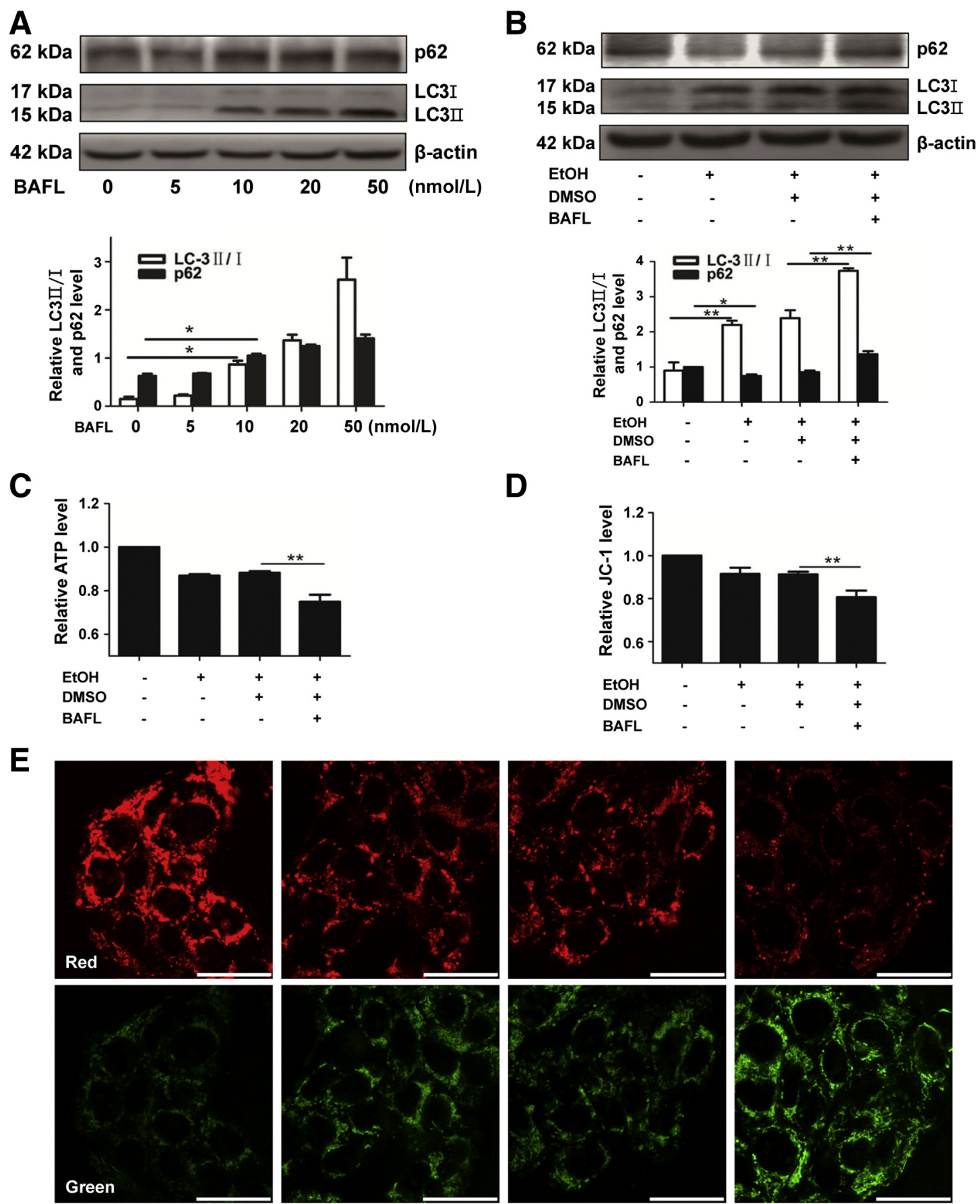

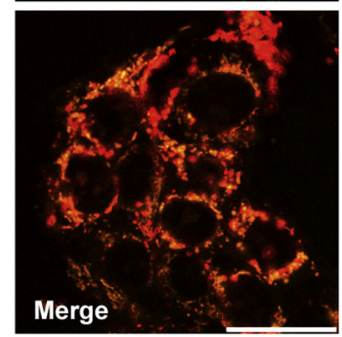

-EtOH

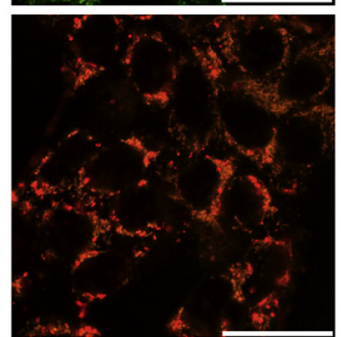

$+\mathrm{EtOH}$

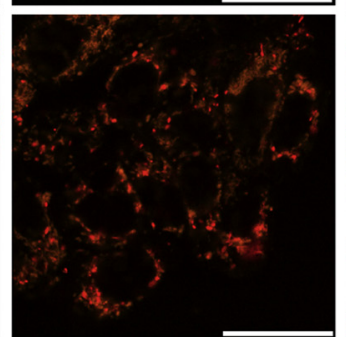

+EtOH+DMSO

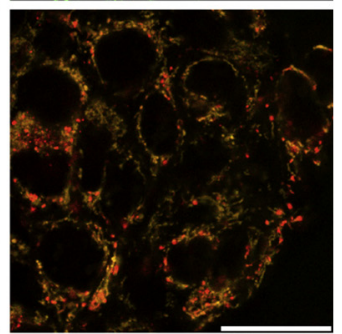

$+\mathrm{EtOH}+\mathrm{BAFL}$

Figure 7 Inhibition of autophagy by bafilomycin A1 (BAFL) attenuates the protective effects of augmenter of liver regeneration. A: LC3 and p62 increase in a dose-dependent manner in the presence of bafilomycin A1. B: Western blot assays show LC3 and p62 expression in the presence of bafilomycin A1. C: Change of ATP in the presence of bafilomycin A1. D and E: Change of mitochondrial transmembrane potential in the presence of bafilomycin A1. The assays were repeated three times. Data are expressed as means \pm SD. ${ }^{*} P<0.05,{ }^{*} P<0.01$. Scale bar $=25 \mu \mathrm{m}$ (E). DMSO, dimethyl sulfoxide; EtOH, ethanol. 
that BafA1 suppressed autophagy in ALR-transfected cells (Figure 7B). Levels of ATP as well as the mitochondrial transmembrane potential (MTP), as visualized by red JC-1 staining in ALR-transfected cells, were reduced after exposure to ethanol in the presence of BafA1, indicating that the protection of hepatocytes against ethanol-induced injury by ALR was at least in part related to autophagy (Figure 7, C-E).

\section{RNA-Seq and Identification of Differentially Expressed Genes}

To further characterize the pathway by which ALR is promoting autophagy, liver transcriptome profile analysis of adenovirus (Ad)-ALR (or $\mathrm{ALR}^{+/-}$) and WT mice was performed in response to ethanol treatment by high-throughput RNA-Seq. On the basis of RNA-Seq, 5046 genes, whose expressions were altered in Ad-ALR mice, and 1171 genes, whose expressions were changed in $\mathrm{ALR}^{+/-}$compared with WT mice, have been identified (Figure 8A). Notably, the phosphatidylinositol 3-kinase-Akt-mTOR pathway was found to be critically involved in Ad-ALR and $\mathrm{ALR}^{+l-}$ mice when compared with WT (Figure 8B), which is consistent with the current understanding of ALR in autophagy. Furthermore, the heatmap analysis revealed that autophagy-related genes, such as DDIT4, were robustly decreased in $\mathrm{ALR}^{+/-}$mice compared with control mice, but clearly increased in Ad-ALR mice (Figure 8C). mTOR kinase complex 1 is a key regulator of autophagy in mammalian cells. Among the essential regulators of mTOR kinase complex 1, DDIT4 (alias REDD1) can rapidly inhibit mTOR kinase complex 1 activity. ${ }^{35,36}$ Further analysis found epidermal growth factor receptor, whose inactivation would induce autophagy, ${ }^{37}$ was lower in Ad-ALR mice. Consequently, the levels of Atg7, Atg10, Atg12, and UNC-51-like autophagy activating kinase 1 were higher in Ad-ALR mice than in WT mice, indicating the activation of autophagy (Figure 8D). On the contrary, Atg7, Atg14, and UNC-51-like autophagy activating kinase 1 were decreased in $\mathrm{ALR}^{+/-}$mice, defining the inhibition of autophagy (Figure 8E). Collectively, these findings suggest that the attenuation of ethanol-induced liver injury by ALR may be achieved through activation of autophagy via the phosphatidylinositol 3-kinase-Akt-mTOR pathway.

\section{ALR Promotion of Autophagy in Ethanol-Induced Liver Injury Is Associated with Suppression of mTOR Signaling}

The correlation between Akt/mTOR pathway and ALR was investigated to understand how ALR rescued ethanolinduced autophagy. The Akt/mTOR signaling pathway is a classic negative regulator of autophagy, and we evaluated the effect of ALR on the activity of mTOR; the results showed that the p-mTOR was down-regulated in ALR-transfected mice compared with the control group (Figure 9A) and upregulated in $\mathrm{ALR}^{+/-}$mice (Figure 9B). Meanwhile, the phosphorylated Akt had no change. Moreover, ethanol treatment also resulted in a significant decrease in p-mTOR in ALR-transfected cells compared with vector-transfected cells (Figure 9C), and the increase of p-mTOR was greater in ALR-knockdown cells than in control cells after ethanol administration (Figure 9D). These results show that the mTOR signaling pathway mediates the effects of ALR on autophagy in response to ethanol treatment.

\section{Discussion}

Alcoholic liver damage is a frequent cause of acute liver failure across the world, but therapeutic options for this fatal disease are limited. ALR is thought to promote hepatocyte survival. Gandhi et $\mathrm{al}^{38}$ showed that deficiency of ALR in mice livers induces mitochondrial degeneration, ATP loss, and cell death, thus exacerbating alcohol-induced hepatotoxicity and liver fibrosis. A study by Kumar et $\mathrm{al}^{18}$ also showed augmented liver injury in ALR knockout mice by alcohol. This study showed that hepatic overexpression of ALR alleviates ethanol-induced liver injury in mice, which is in line with its ability to protect the liver from a variety of other toxic insults. Furthermore, the protection conveyed by ALR expression may be mediated by promoting mitophagy and activating the mTOR signaling pathway. The results confirmed an association of ALR overexpression with increased LC3II conversion and p62 degradation both in vivo and in vitro. Compared with control cells, ALR overexpression also up-regulated Atg5 and beclin-1 expression and increased Parkin translocation to mitochondria after ethanol stimulation. These results indicate that ALR induction enhances autophagy and mitophagy in response to ethanol-induced liver injury.

Autophagy plays an important role in both normal and pathologic conditions. Although mitophagy selectively removes damaged mitochondria to protect against ROS generation, protecting liver from oxidative stress and cell death, ${ }^{39}$ mitochondrial protection still becomes the most priority issue to be considered during alcoholic hepatotoxicity. It has been reported that in alcohol-induced liver disease, autophagy is a protective mechanism against the liver injury caused by alcohol. ${ }^{40}$ Parkin is one of the important genes to promote mitophagy, and deletion of this Parkin gene in mice caused liver mitochondria less capable to adapt to Gao-binge ethanol treatment compared with wildtype mice. ${ }^{41}$ Furthermore, treatment of ethanol-induced liver injury with rapamycin alleviated hepatic injury by enhancing autophagy in hepatocytes in vitro and in vivo. ${ }^{12}$ In this study, ALR significantly increased the expression of autophagy-related proteins and ameliorated alcoholinduced mitochondrial dysfunction, consistent with a possible therapeutic effect of ALR on ethanol-induced liver injury. Although ALR has been demonstrated to protect liver from various liver injuries, the characterization of its role against acute alcoholic liver injury via promoting 

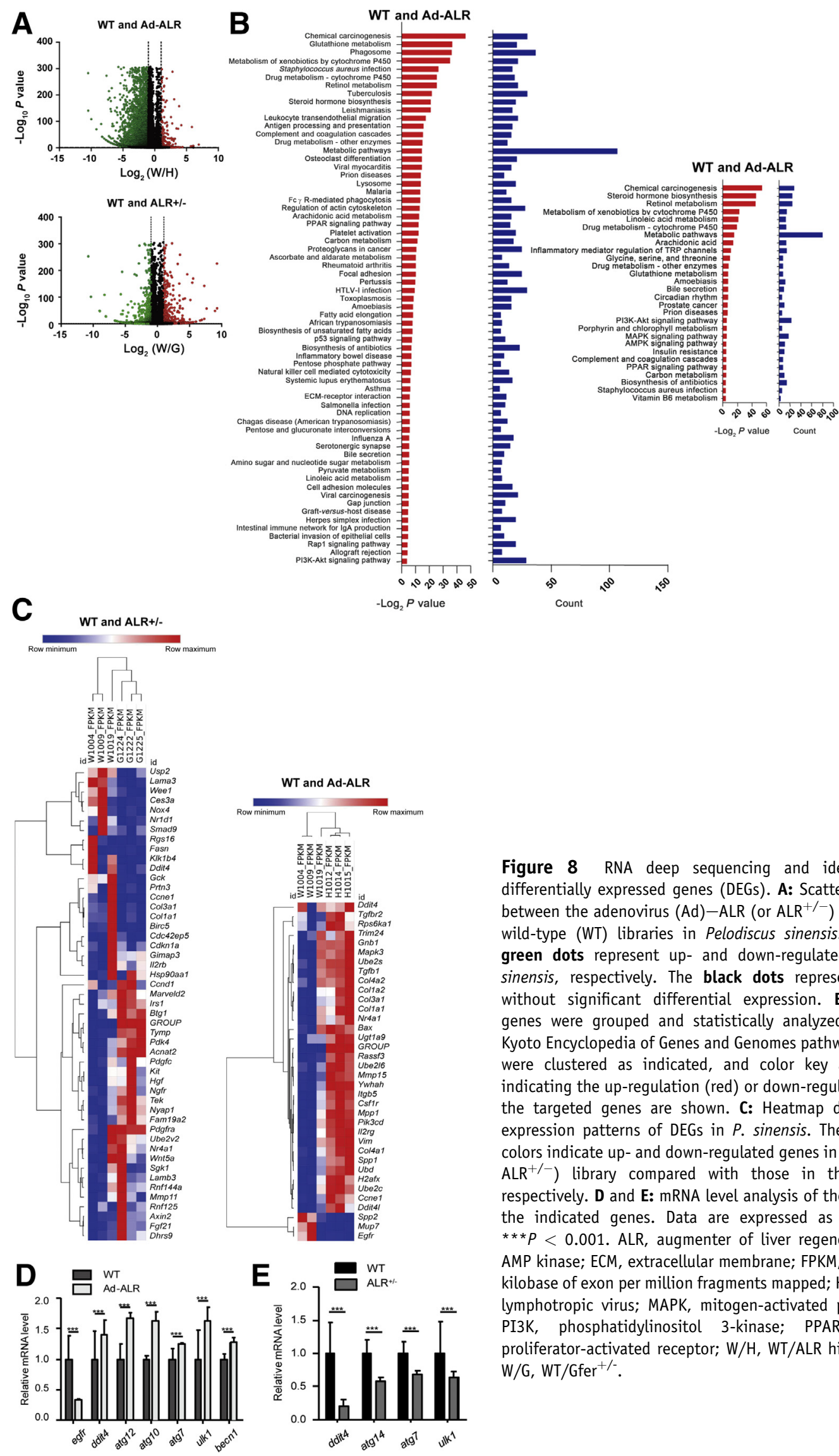

Figure 8 RNA deep sequencing and identification of differentially expressed genes (DEGs). A: Scatter plot of DEGs between the adenovirus (Ad)-ALR (or $\mathrm{ALR}^{+/-}$) library and the wild-type (WT) libraries in Pelodiscus sinensis. The red and green dots represent up- and down-regulated genes in $P$. sinensis, respectively. The black dots represent the genes without significant differential expression. B: Coregulated genes were grouped and statistically analyzed according to Kyoto Encyclopedia of Genes and Genomes pathways. The genes were clustered as indicated, and color key and histogram indicating the up-regulation (red) or down-regulation (blue) of the targeted genes are shown. C: Heatmap diagram of the expression patterns of DEGs in $P$. sinensis. The red and blue colors indicate up- and down-regulated genes in the Ad-ALR (or $\mathrm{ALR}^{+/-}$) library compared with those in the WT library, respectively. D and E: mRNA level analysis of the expression of the indicated genes. Data are expressed as means \pm SD. ${ }^{*} * P<0.001$. ALR, augmenter of liver regeneration; AMPK, AMP kinase; ECM, extracellular membrane; FPKM, fragments per kilobase of exon per million fragments mapped; HTLV, human Tlymphotropic virus; MAPK, mitogen-activated protein kinase; PI3K, phosphatidylinositol 3-kinase; PPAR, peroxisome proliferator-activated receptor; W/H, WT/ALR high expression; W/G, WT $/ \mathrm{Gfer}^{+/}$. 
A
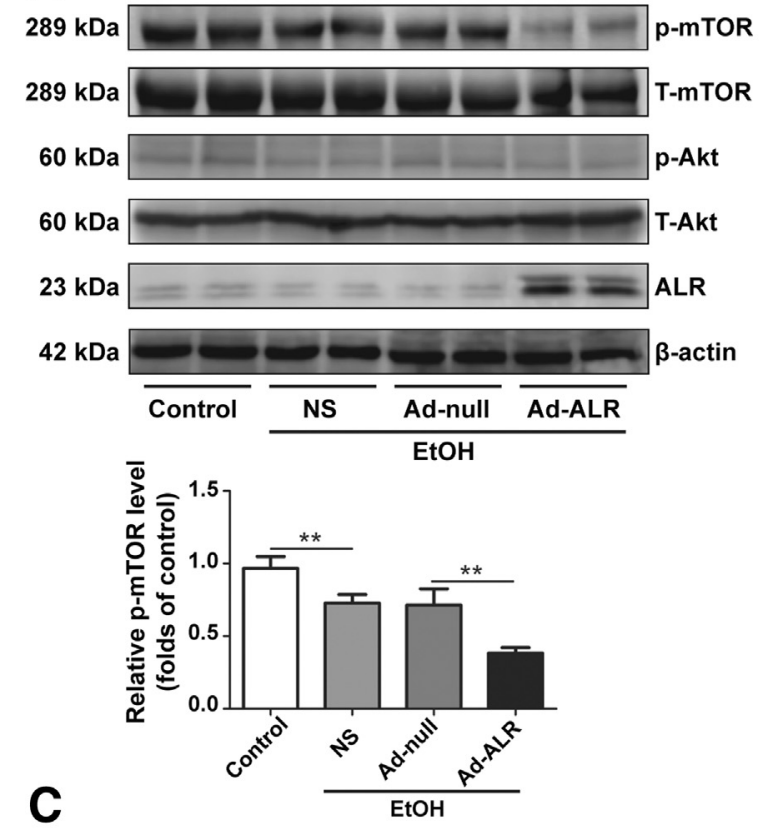

B

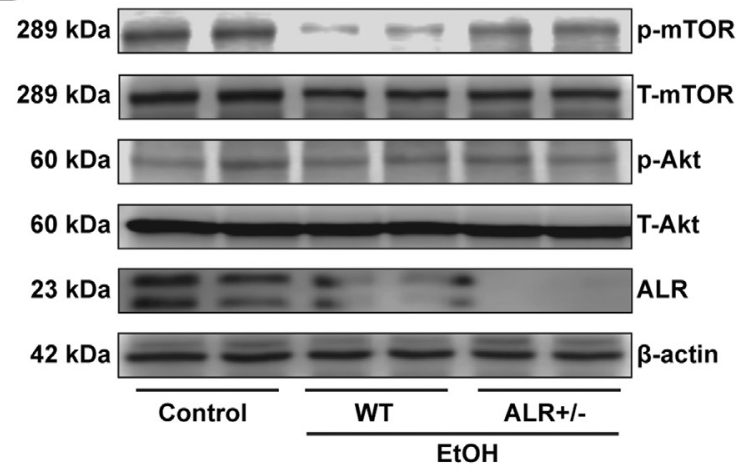

D
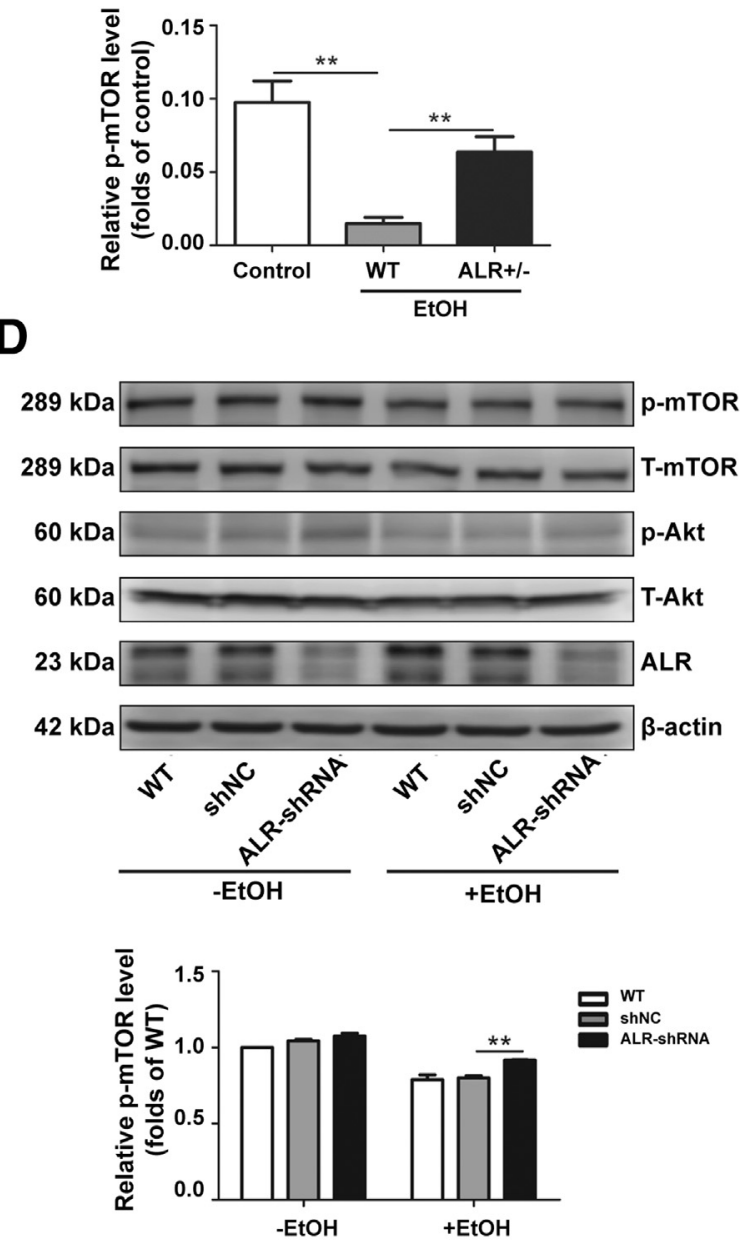

Figure 9 Analysis of the activity of Akt/mammalian target of rapamycin (mTOR) signaling pathway in mouse livers and cells when overexpressing augmenter of liver regeneration (ALR) or ALR knockdown after ethanol (EtOH) exposure. Western blot analyses were performed to detect phosphorylated mTOR (p-mTOR) or phosphorylated Akt (p-Akt) in Ad-ALR mice or ALR ${ }^{+/-}$mice (A and $\left.\mathbf{B}\right)$ and in ALR-transfected cells or ALR deletion cells (C and $\left.\mathbf{D}\right)$. All data were repeated three times. Data are expressed as means \pm SD. ${ }^{*} P<0.05,{ }^{*} P<0.01$. NS, normal saline; shNC, negative control; $T$, total; Tx, treatment; WT, wild type.

mitophagy will expand our viewing this liver protector at new sight.

Our speculation regarding the role of autophagy is supported by the observation that the protective effect of ALR can be attenuated by cotreatment with bafilomycin A1. ALR liver protection is closely related to Akt signaling pathway ${ }^{42}$; among signaling pathways involved in autophagy, phosphatidylinositol 3-kinase/Akt-mTOR pathway is widely acceptable. mTOR is a regulator of cell growth, proliferation, autophagy, and other physiological processes. ${ }^{27}$ mTOR regulates autophagy negatively by phosphorylation of ATG13, which reduces its interaction with UNC-51-like autophagy activating kinase 1 and inhibits the formation of the trimeric UNC-51-like autophagy 
activating kinase 1 kinase complex required for autophagosome formation. ${ }^{43}$ Studies have shown that downregulation of mTOR activity triggers autophagy, ${ }^{44,45}$ which is in line with our speculation regarding mechanisms of ALR-induced autophagy. This study confirms that the increase in p-mTOR expression after ethanol exposure is inhibited by ALR administration. However, ALR seems not to affect the expression of upstream signal Akt after ethanol exposure. The results highly suggest that ALR promotes autophagy via an mTOR-dependent pathway.

In conclusion, ALR protected the liver from ethanol injury by enhancing autophagy, and the protection was mediated by mTOR signaling. Further studies are required to depict the interaction of ALR with mTOR in greater detail, with the aim of identifying targets for pharmacologic intervention to mimic the protective effects mediated by ALR overexpression. Considering the enormous global cost of alcoholic liver damage in terms of money and human suffering, a closer investigation into liver-specific enhancement of mitophagy as a strategy for pharmacologic amelioration of alcoholic liver disease appears a promising and worthwhile goal. Our results suggest a possibility that extragenously delivered ALR gene could provide potential clinical therapy to combat with alcoholic liver disease. Nevertheless, the identification of ALR as a novel inducer of autophagy may not only serve as a new lead for developing new therapeutic agents for alcoholic liver disease, it may additionally serve as a novel molecular probe for studying the regulation of autophagy.

\section{Acknowledgments}

We thank Xin Wang, Dr. Xiaowei Jia, Dr. Jing Zhang, and Dr. Weichun Xiao for help in experiments and proofreading of the manuscript.

\section{References}

1. Rehm J, Samokhvalov AV, Shield KD: Global burden of alcoholic liver diseases. J Hepatol 2013, 59:160-168

2. Gao B, Bataller R: Alcoholic liver disease: pathogenesis and new therapeutic targets. Gastroenterology 2011, 141:1572-1585

3. Williams JA, Manley S, Ding WX: New advances in molecular mechanisms and emerging therapeutic targets in alcoholic liver diseases. World J Gastroenterol 2014, 20:12908-12933

4. Cederbaum AI, Lu Y, Wu D: Role of oxidative stress in alcoholinduced liver injury. Arch Toxicol 2009, 83:519-548

5. Higuchi H, Kurose I, Kato S, Miura S, Ishii H: Ethanol-induced apoptosis and oxidative stress in hepatocytes. Alcohol Clin Exp Res 1996, 20:340A-346A

6. Ding WX, Manley S, Ni HM: The emerging role of autophagy in alcoholic liver disease. Exp Biol Med 2011, 236:546-556

7. Ding WX, Yin XM: Mitophagy: mechanisms, pathophysiological roles, and analysis. Biol Chem 2012, 393:547-564

8. Youle RJ, Narendra DP: Mechanisms of mitophagy. Nat Rev Mol Cell Biol 2011, 12:9-14

9. Lemasters JJ: Variants of mitochondrial autophagy: types 1 and 2 mitophagy and micromitophagy (type 3). Redox Biol 2014, 2:749-754
10. Ding WX, Li M, Yin XM: Selective taste of ethanol-induced autophagy for mitochondria and lipid droplets. Autophagy 2011, 7: 248-249

11. Ding WX, Li M, Chen X, Ni HM, Lin CW, Gao W, Lu B, Stolz DB, Clemens DL, Yin XM: Autophagy reduces acute ethanol-induced hepatotoxicity and steatosis in mice. Gastroenterology 2010, 139:1740-1752

12. Lin CW, Zhang H, Li M, Xiong X, Chen X, Chen X, Dong XC, Yin XM: Pharmacological promotion of autophagy alleviates steatosis and injury in alcoholic and non-alcoholic fatty liver conditions in mice. J Hepatol 2013, 58:993-999

13. LaBrecque DR, Pesch LA: Preparation and partial characterization of hepatic regenerative stimulator substance (SS) from rat liver. J Physiol 1975, 248:273-284

14. Mei MH, An W, Zhang BH, Shao Q, Gong DZ: Hepatic stimulator substance protects against acute liver failure induced by carbon tetrachloride poisoning in mice. Hepatology 1993, 17:638-644

15. Ho DW, Fan ST, To J, Woo YH, Zhang Z, Lau C, Wong J: Selective plasma filtration for treatment of fulminant hepatic failure induced by D-galactosamine in a pig model. Gut 2002, 50:869-876

16. Wu Y, Chen L, Yu H, Liu H, An W: Transfection of hepatic stimulator substance gene desensitizes hepatoma cells to $\mathrm{H}_{2} \mathrm{O}_{2}$-induced cell apoptosis via preservation of mitochondria. Arch Biochem Biophys 2007, 464:48-56

17. Tzirogiannis KN, Panoutsopoulos GI, Demonakou MD, Hereti RI, Alexandropoulou KN, Mykoniatis MG: Effect of hepatic stimulator substance (HSS) on cadmium-induced acute hepatotoxicity in the rat liver. Dig Dis Sci 2004, 49:1019-1028

18. Kumar S, Wang J, Rani R, Gandhi CR: Hepatic deficiency of augmenter of liver regeneration exacerbates alcohol-induced liver injury and promotes fibrosis in mice. PLoS One 2016, 11: e0147864

19. Lange H, Lisowsky T, Gerber J, Muhlenhoff U, Kispal G, Lill R: An essential function of the mitochondrial sulfhydryl oxidase Erv1p/ALR in the maturation of cytosolic Fe/S proteins. EMBO Rep 2001, 2: $715-720$

20. Wu Y, Zhang J, Dong L, Li W, Jia J, An W: Hepatic stimulator substance mitigates hepatic cell injury through suppression of the mitochondrial permeability transition. FEBS J 2010, 277: 1297-1309

21. Thirunavukkarasu C, Wang LF, Harvey SA, Watkins SC, Chaillet JR, Prelich J, Starzl TE, Gandhi CR: Augmenter of liver regeneration: an important intracellular survival factor for hepatocytes. J Hepatol 2008, 48:578-588

22. Committee for the Update of the Guide for the Care and Use of Laboratory Animals: National Research Council: Guide for the Care and Use of Laboratory Animals: Eighth Edition. Washington, DC, National Academies Press, 2011

23. Ren S, Peng Z, Mao JH, Yu Y, Yin C, Gao X, Cui Z, Zhang J, Yi K, Xu W, Chen C, Wang F, Guo X, Lu J, Yang J, Wei M, Tian Z, Guan Y, Tang L, Xu C, Wang L, Gao X, Tian W, Wang J, Yang H, Wang J, Sun Y: RNA-seq analysis of prostate cancer in the Chinese population identifies recurrent gene fusions, cancer-associated long noncoding RNAs and aberrant alternative splicings. Cell Res 2012, 22: $806-821$

24. Love MI, Hogenesch JB, Irizarry RA: Modeling of RNA-seq fragment sequence bias reduces systematic errors in transcript abundance estimation. Nat Biotechnol 2016, 34:1287-1291

25. Trapnell C, Roberts A, Goff L, Pertea G, Kim D, Kelley DR, Pimentel H, Salzberg SL, Rinn JL, Pachter L: Differential gene and transcript expression analysis of RNA-seq experiments with TopHat and Cufflinks. Nat Protoc 2012, 7:562-578

26. Huang DW, Sherman BT, Lempicki RA: Systematic and integrative analysis of large gene lists using DAVID bioinformatics resources. Nat Protoc 2009, 4:44-57

27. Huang DW, Sherman BT, Lempicki RA: Bioinformatics enrichment tools: paths toward the comprehensive functional analysis of large gene lists. Nucleic Acids Res 2009, 37:1-13 
28. Mizushima N, Yoshimori T, Levine B: Methods in mammalian autophagy research. Cell 2010, 140:313-326

29. Itakura E, Mizushima N: Characterization of autophagosome formation site by a hierarchical analysis of mammalian Atg proteins. Autophagy 2010, 6:764-776

30. Yan L, Yang H, Li Y, Duan H, Wu J, Qian P, Li B, Wang S: Regulator of calcineurin 1-1L protects cardiomyocytes against hypoxia-induced apoptosis via mitophagy. J Cardiovasc Pharmacol 2014, 64:310-317

31. Narendra D, Tanaka A, Suen DF, Youle RJ: Parkin is recruited selectively to impaired mitochondria and promotes their autophagy. J Cell Biol 2008, 183:795-803

32. Yoshimori T, Yamamoto A, Moriyama Y, Futai M, Tashiro Y: Bafilomycin A1, a specific inhibitor of vacuolar-type $\mathrm{H}(+)$-ATPase, inhibits acidification and protein degradation in lysosomes of cultured cells. J Biol Chem 1991, 266:17707-17712

33. Bowman EJ, Siebers A, Altendorf K: Bafilomycins: a class of inhibitors of membrane ATPases from microorganisms, animal cells, and plant cells. Proc Natl Acad Sci U S A 1988, 85:7972-7976

34. Ohkuma S, Shimizu S, Noto M, Sai Y, Kinoshita K, Tamura H: Inhibition of cell growth by bafilomycin A1, a selective inhibitor of vacuolar $\mathrm{H}(+)-$ ATPase: in vitro cell. In Vitro Cell Dev Biol Anim 1993, 29A:862-866

35. Sofer A, Lei K, Johannessen CM, Ellisen LW: Regulation of mTOR and cell growth in response to energy stress by REDD1. Mol Cell Biol 2005, 25:5834-5845

36. Qiao S, Dennis M, Song X, Vadysirisack DD, Salunke D, Nash Z, Yang Z, Liesa M, Yoshioka J, Matsuzawa S, Shirihai OS, Lee RT, Reed JC, Ellisen LW: A REDD1/TXNIP pro-oxidant complex regulates ATG4B activity to control stress-induced autophagy and sustain exercise capacity. Nat Commun 2015, 6:7014

37. Wei Y, Zou Z, Becker N, Anderson M, Sumpter R, Xiao G, Kinch L, Koduru P, Christudass CS, Veltri RW, Grishin NV, Peyton M, Minna J, Bhagat G, Levine B: EGFR-mediated Beclin 1 phosphorylation in autophagy suppression, tumor progression, and tumor chemoresistance. Cell 2013, 154:1269-1284

38. Gandhi CR, Chaillet JR, Nalesnik MA, Kumar S, Dangi A, Demetris AJ, Ferrell R, Wu T, Divanovic S, Stankeiwicz T, Shaffer B, Stolz DB, Harvey SA, Wang J, Starzl TE: Liver-specific deletion of augmenter of liver regeneration accelerates development of steatohepatitis and hepatocellular carcinoma in mice. Gastroenterology 2015, 148:379-391

39. Lee J, Giordano S, Zhang J: Autophagy, mitochondria and oxidative stress: cross-talk and redox signalling. Biochem J 2012, 441:523-540

40. Wang L, Khambu B, Zhang H, Yin X: Autophagy in alcoholic liver disease, self-eating triggered by drinking. Clin Res Hepatol Gastroenterol 2015, 39:s2-s6

41. Williams JA, Ni HM, Ding Y, Ding WX: Parkin regulates mitophagy and mitochondrial function to protect against alcohol-induced liver injury and steatosis in mice. Am J Physiol Gastrointest Liver Physiol 2015, 309:G324-G340

42. Guo YY, Wu Y, Jia XW, An W: Augmenter of liver regeneration potentiates doxorubicin anticancer efficacy by reducing the expression of ABCB1 and ABCG2 in hepatocellular carcinoma. Lab Invest 2017, 97:1400-1411

43. Jung $\mathrm{CH}$, Jun $\mathrm{CB}$, Ro SH, Kim YM, Otto NM, Cao J, Kundu M, Kim DH: ULK-Atg13-FIP200 complexes mediate mTOR signaling to the autophagy machinery. Mol Biol Cell 2009, 20:1992-2003

44. Matsuzawa Y, Oshima S, Takahara M, Maeyashiki C, Nemoto Y, Kobayashi M, Nibe $Y$, Nozaki $K$, Nagaishi T, Okamoto R, Tsuchiya K, Nakamura T, Ma A, Watanabe M: TNFAIP3 promotes survival of CD4 T cells by restricting MTOR and promoting autophagy. Autophagy 2015, 11:1052-1062

45. Pattingre S, Espert L, Biard-Piechaczyk M, Codogno P: Regulation of macroautophagy by mTOR and Beclin-1 complexes. Biochimie 2008, 90:313-323 